Research article

\title{
PKQuest: a general physiologically based pharmacokinetic model. Introduction and application to propranolol David G Levitt
}

Address: Department of Physiology, 6-125 Jackson Hall, 321 Church St. S. E., Minneapolis, MN 55455, USA

E-mail: levitt@dcmir.med.umn.edu

Published: 15 August 2002

BMC Clinical Pharmacology 2002, 2:5

This article is available from: http://www.biomedcentral.com/1472-6904/2/5

(C) 2002 Levitt; licensee BioMed Central Ltd. This article is published in Open Access: verbatim copying and redistribution of this article are permitted in all media for any non-commercial purpose, provided this notice is preserved along with the article's original URL.

\begin{abstract}
Background: A "physiologically based pharmacokinetic" (PBPK) approach uses a realistic model of the animal to describe the pharmacokinetics. Previous PBPKs have been designed for specific solutes, required specification of a large number of parameters and have not been designed for general use.

Methods: This new PBPK program (PKQuest) includes a "Standardhuman" and "Standardrat" data set so that the user input is minimized. It has a simple user interface, graphical output and many new features: 1) An option that uses the measured plasma concentrations to solve for the time course of the gastrointestinal, intramuscular, intraperotineal or skin absorption and systemic availability of a drug - for a general non-linear system. 2) Capillary permeability limitation defined in terms of the permeability-surface area products. 4) Saturable plasma and tissue protein binding. 5) A lung model that includes perfusion-ventilation mismatch. 6) A general optimization routine using either a global (simulated annealing) or local (Powell) minimization applicable to all model parameters.
\end{abstract}

Results: PKQuest was applied to measurements of human propranolol pharmacokinetics and intestinal absorption. A meal has two effects: I) increases portal blood flow by $50 \%$; and 2 ) decreases liver metabolism by $20 \%$. There is a significant delay in the oval propranolol absorption in fasting subjects that is absent in fed subjects. The oral absorption of the long acting form of propranolol continues for a period of more than 24 hours.

Conclusions: PKQuest provides a new general purpose, easy to use, freely distributed [www.pkquest.com] and physiologically rigorous PBPK software routine.

\section{Background}

The expression "physiologically based pharmacokinetic model" (PBPK) describes an approach where the drug kinetics are modeled using a realistic physiological description of the animal. This has now become a standard approach to pharmacokinetics and has been applied to many different compounds [1]. Despite this, most human drug studies still rely on approximate compartmental pharmacokinetic techniques and do not attempt a complete physiological model. One reason for this is that detailed kinetic information for the different organs is not available in humans to the same extent as it is in animal studies. A second reason is that PBPK models are still somewhat difficult to use and remain a tool primarily of specialists. 
This paper, along with the accompanying papers [2-4], describes a new PBPK modeling program (PKQuest) that is designed to overcome both of these problems. The first problem (lack of information) is avoided by preprogramming a large set of data about kinetics in different organs that can be called as default variables applicable to whatever solute is being investigated. The second problem (ease of use) is overcome by using a simple interface and a graphical and printed output that provides a complete description of the pharmacokinetics. Despite this ease of use, PKQuest provides a rigorously accurate PBPK applicable to all the different physiological and biochemical properties of the different compounds that are commonly investigated.

This first paper introduces the general features of the program, describes the mathematical details and explains the simple interface that has been designed to use it. PKQuest has incorporated a large number of features, some of which have been developed and introduced for the first time (see below). Some of these features are illustrated in this paper by using PKQuest to obtain new information about the human pharmacokinetics of propranolol. This is an intensively investigated area. For example, a search in PubMed for the terms "propranolol" AND "pharmacokinetics" yielded 1306 references. Despite this, there are still several important questions remaining about its behavior in man. In particular, the rather peculiar effect of food intake on plasma levels remains unexplained [5]: Meals decrease the plasma levels when propranolol is given intravenously (IV), and increase the plasma levels when propranolol is given orally (PO). Since it has been shown that gastrointestinal absorption is complete [6], this meal effect must result from a food induced change in the metabolism, distribution or rate of absorption. Since liver propranolol metabolism is partially limited by liver blood flow [7], the meal induced decrease in the plasma level of the IV administered propranolol can be explained by the increase in liver blood flow that has been shown to be associated with a meal [5,8-10]. However, this would not explain the meal induced increase in plasma levels following the oral propranolol. PKQuest will be used to try and provide an explanation of this behavior. This analysis is based primarily on the experimental data of Olanoff et. al. [5] in which the plasma propranolol levels were measured after simultaneous IV and PO administration, eliminating the effects of individual variation from these measurements. Requiring a single set of parameters to fit both the simultaneous IV and PO plasma concentration data imposes a strong limitation on the model. Comparing the PBPK parameter set for the fed and fasted case then provides quantitative information about the effect of meals on propranolol pharmacokinetics. This analysis makes extensive use of the novel PKQuest feature that uses the blood levels following an oral dose to calculate the time course of the intestinal PO absorption.

In the last section, PKQuest will be used to quantitate the time course of intestinal absorption of a sustained release form of propranolol.

The modeling program, along with the detailed applications described in these papers, is freely available on the Web at [www.pkquest.com].

\section{Methods \\ Flow diagram}

PKQuest uses the conventional PBPK flow diagram [1] shown in fig. 1. Each box corresponds to a well-mixed compartment and each arrow is an input or output to the compartment determined by the blood flow. The different compartments are numbered from 0 (vein) to 11 (lipid). Each compartment is described by the total organ blood flow (liters/min) and the total weight (Kg). In PKQuest the names shown in fig. 1 can be used in place of the numbers. For example the muscle blood flow can be described either by F[7] or F [muscle]. Table 1I lists the values of organ weight and the blood flow per organ weight for each organ for the standard human $(70 \mathrm{Kg})$. These organ weights are for the default value of $20 \%$ body fat. They are scaled depending on the value of "Fat" that is input. The "portal" compartment corresponds to all the organs drained by the portal vein (stomach, small and large intestine, pancreas and spleen) with a blood flow equal to that in the portal vein. The "liver" blood flow corresponds to the hepatic artery. The compartment labeled "other" groups all the other organs with a flow and weight adjusted to account for the total body weight $(70 \mathrm{Kg})$ and the total default cardiac output (6.06 liters/min). For a complete listing of all parameters and constants used in PKQuest, download the file gensolve.mws from the PKQuest web site [www.pkquest.com] and view the routines "standardman()" and "defaultpar()".

A number of other properties are associated with each compartment such as the water volume fraction of the tissue (wfract, liters/Kg), the fraction of the compartment water that is extracelluar (ecf), the capillary clearance (flcear), etc. The critical variable describing the kinetics is the free water concentration c[i] for each compartment $i$. This is the concentration that determines the rate of exchange across the capillary. It is related to the experimental concentration C[i] (amount/Kg) by the parameter fw[i] which is the fraction of the total solute in compartment $i$ that is free in the water phase $\left(c=f w^{*} C / w f r a c t\right)$. The parameter $\mathrm{fw}$ is determined by factors such as protein binding and lipid solubility. Although fw is the parameter used in the actual calculations, its value is usually input in terms of other parameters, such as the lipid/water parti- 


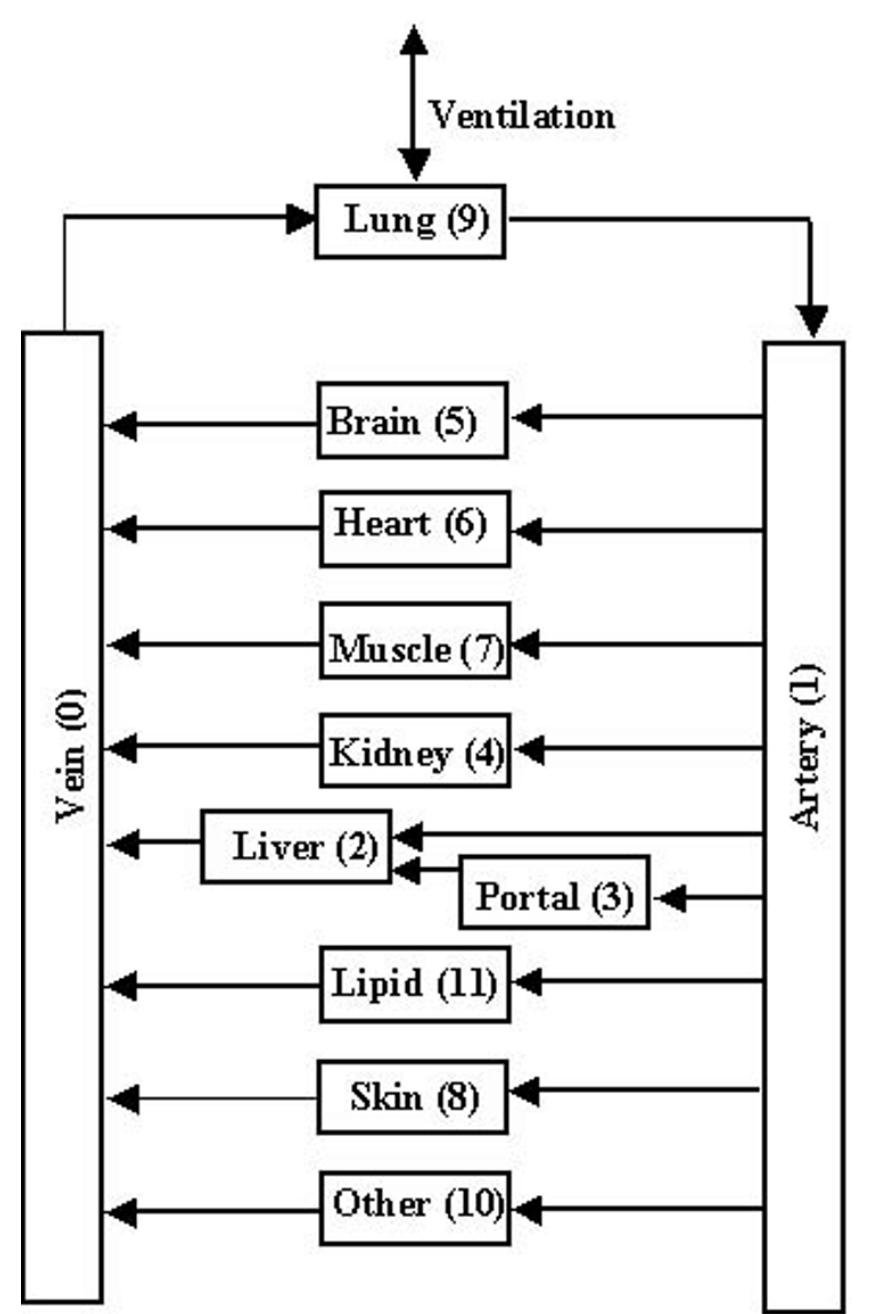

Figure I

Flow diagram used for PBPK model. Each box represents a well-stirred compartment and each arrow is an input or output to the organ.

tion coefficient [2] or the protein binding [4]. For the propranolol calculations, $f w[i]$ is related to the input parameters ktiss[i] (the ratio of tissue to plasma concentration) and freepl (the fraction free in plasma) by the relation: $\mathrm{fw}[\mathrm{i}]=$ freepl $^{*}$ watfr[i]/(watfrpl*ktiss[i]) where watfr[i] and watfrpl are the water fractions in tissue $i$ and in the plasma.

Each compartment can have a metabolic component (Q) described either by the Michaelis-Menten parameters Vm and $\mathrm{Km}\left(\mathrm{Q}[\mathrm{i}]=\mathrm{Vm}[\mathrm{i}]^{*} \mathrm{c}[\mathrm{i}] /(\mathrm{Km}[\mathrm{i}]+\mathrm{c}[\mathrm{i}])\right)$ if the metabolism saturates or by a metabolic clearance (Tclr) if the metabolism is linear. The kidney is a special case, removing solute either via filtered renal clearance (rclr) or by tubule secretion (described by Vm[kidney], Km[kidney] and Tclr[kidney]). (The default is no tubule secretion or clearance). Each compartment can also have its own unique in-
Table I: Organ Weights and Blood Flows for Standard Human (70 Kg, 20\% Fat)

\begin{tabular}{lll}
\hline Organ & Weight $\mathbf{K g})$ & $\begin{array}{l}\text { Blood Flow } \\
\text { (lit/min/Kg) }\end{array}$ \\
\hline Artery & & \\
Vein & 1.1 & --- \\
Liver & 4.4 & --- \\
& 1.8 & 0.25 (hepatic \\
Portal & 1.5 & artery) \\
& & 0.75 (portal \\
Kidney & 0.31 & vein) \\
muscle & 33.0 & 4.0 \\
brain & 1.4 & 0.0266 \\
heart & 0.33 & 0.56 \\
lung & 0.536 & 0.8 \\
skin & 2.6 & 14.98 \\
fat & 14 & 0.12 \\
"other" & 4.12 & 0.056 \\
"bone" & 4.90. & 0.054 \\
& & 0.0 \\
& &
\end{tabular}

put from outside the body (cinput[i]). The standard inputs are to the vein, lung or GI tract. It is assumed that the PO absorption goes directly into the liver via the portal vein. This choice was made (as opposed to PO input into the portal compartment) because the solute absorbed by, e.g., the small intestine is picked up by the villous capillaries and removed in the portal vein before it distributes in the entire small intestine or even in the local intestinal submucosal tissue compartment.

It is assumed that the tissue region of each organ can be modeled as a single well-stirred compartment. This is clearly an approximation and effects such as diffusion gradients, countercurrent exchange and heterogeneous organ blood flows [11-13] will produce small deviations from this assumption. The exchange between the vascular and tissue compartments results from diffusion of the free solute between the blood and this well mixed tissue region during the passage through the capillary (see Appendix, fig. 7). The rate of this exchange is determined by the capillary permeability-surface area product (PS), which can be related to the parameter fclear (see Appendix, eq. 3), which varies from 0 (impermeable) to 1 (flow limited). For flow limited solutes (e.g. propranolol), the free water concentration (c) in the vein leaving the tissue is equal to the free tissue water concentration.

The major limitation of this model is the representation of the venous and arterial vascular spaces as single compartments. This means that PKQuest cannot provide an accurate picture of the very early time course of drug 
distribution (less than a minute) when a more detailed model of the vascular compartments is required [14].

\section{Determination of physiological parameters}

The most important feature of a PBPK is the accuracy and validity of the physiological parameters (organ weight, blood flow, volume of distribution, etc.). The major advantage of a PBPK over a simpler 2 or 3 compartment model is that, presumably, it returns a higher resolution view of the way the body handles the drug. This presumption depends on the assumption that the physiological parameters in the model are correct. A large amount of effort has been expended on the choice of these parameters in PKQuest. Initially, the parameters were chosen using standard references, such as the "Report on the Task Group on Reference Man" [15] and "Reference values for resting blood flow to organs of man" [16] and by copying values used from previous PBPK models [1,17]. Then the parameters were adjusted to insure that they were in agreement with such features as total blood volume and cardiac output $[18,19]$, extracellular space [20] and total body water $[21,22]$. Finally, certain parameters were adjusted to optimize the fit to the pharmacokinetics of selected solutes. For example, muscle blood flow was adjusted to fit the $\mathrm{D}_{2} \mathrm{O}$ kinetics [3], fat blood flow to fit the enflurane kinetics [2], and total extracellular space to fit the inulin kinetics [4]. All of the human physiological parameters are listed in the "standardhuman" procedure in the PKQuest program.

Since muscle represents about $50 \%$ of the total water volume, the muscle blood flow and volume are the two most important parameters in the PBPK. The value of the muscle blood flow would be expected to have large variations depending on whether, for example, the subject is lying on a couch with an IV catheter in his arm or is ambulatory after an oral drug dose. It is a gross approximation to treat all the muscles as a single compartment with a single blood flow and some PBPKs have divided the muscle into a "working" and "resting" compartment [23]. However, sub-division of the muscle compartment increases the complexity of the model beyond what is usually justified by the available data. For this reason, the muscle blood flow is the parameter that is most frequently varied for the different studies described in these papers and will be regarded as an adjustable parameter. In PKQuest, when the blood flow of one organ is changed, all the other organ flows remain the same, so that the total cardiac output is also changed. The default value for the muscle blood flow is at the low end of most estimates (0.0266 liters/Kg), representing $14 \%$ of the cardiac output. In several of the studies described in these papers, the muscle blood flow has been increased by up to a factor of two.
The focus in this series of papers is on human pharmacokinetics (using the "standardhuman" parameter set). In addition, a "standardrat" parameter set is included in the current version of PKQuest. The physiological parameters for this rat data set have been chosen and refined using the same approach that was described here for the human data set.

\section{Implementation}

PKQuest is written using the Maple (version 6, 7 or 8) computer algebra system [www.maplesoft.com]. This means that the user must have Maple installed and running before PKQuest can be used. This limitation is outweighed by the advantage of being able to use all of the features that are preprogrammed in Maple, such as the numerical solution and graphical options. Maple is available for most operating systems (PC, Mac, Linux) and PKQuest should run on any system that has Maple installed.

The derivations and mathematic details of the modeling program are described in the Appendix. PKQuest is so large and has so many optional features that not all of them can be described here. The Appendix focuses on the features of the program that are new and have not previously been incorporated into a PBPK model, including the features 4, 5, 6, 7 and 8 listed below. The description of many other features is available using the Maple "help" option that has been programmed into PKQuest. This allows the user to enter the name of a term or parameter that is used in the program and view a description of the feature and how it should be used. As a typical example, entering the term fclear in the help option pops up a help page that begins:

fclear [i] is measure of capillary permeability:

fclear $[i]=$ first pass clearance of solute $=($ Cartery Cvein)/(Cartery-Ctissue) where C's are free water concentrations.

It is related to the capillary permeability by: fclear $=1-\exp \left(-f w[\text { artery }]^{*} p s / F\right)$ for organ $i$ where $p=$ capillary permeability, $s=$ capillary surface area, $F=$ blood flow, and fw [artery $]=$ fraction of solute that is free (unbound) in arterial blood.

NOTE that the effective permeability is the product of the fraction free in blood ( $f w$ [artery]) times the classical permeability. For cases where fw [artery] is very small, the solute can become diffusion limited even for solutes with a large capillary ps.

Flow limited case (Cvein=Ctissue): fclear $[i]=1$ (=maximum allowed value) 
This is the first third of this help page. It goes on to describe the different options for entering values of fclear[i] and for varying and optimizing this parameter. Almost every parameter in PKQuest has a similar help entry.

PKQuest is a large program, consisting of more than 30 separate Maple procedures and a number of Maple worksheets. Once the program has been installed, the user only needs to deal with two simple procedures, each of which is contained in one worksheet: The first procedure lists the values of the model parameters that differ from the default values for the specific solute that is being investigated. This procedure is labeled with a name that describes the compound being investigated. The second procedure calls the data in the first procedure, runs the program and determines the type of output that is displayed. In the remainder of this paper, the use of these two procedures will be illustrated by an investigation of the human propranolol pharmacokinetics.

\section{Experimental data}

Three sets of experimental data were used. The first set was from the paper of Fagan et. al. [24]. It described measurements of the plasma propranolol concentration from 1 minute to 8 hours after a $0.05 \mathrm{mg} / \mathrm{Kg}$ dose infused at a rate of $1 \mathrm{mg} / \mathrm{min}$ in 6 young normal males (weight $60-90 \mathrm{Kg}$ ). The second set was the data of Olanoff et. al. [5] for the simultaneous IV infusion of $0.1 \mathrm{mg} / \mathrm{Kg}$ (infused at a rate of $1 \mathrm{mg} / \mathrm{min}$ ) of unlabeled racemic propranolol and oral dose of $80 \mathrm{mg}$ (consisting of $40 \mathrm{mg}$ of the hexadeuteriumlabeled (+)-propranolol hydrochloride and $40 \mathrm{mg}$ of the dideuterium-labeled (-) propranolol hydrochloride). The subjects were 6 healthy males weighing 70 to $100 \mathrm{Kg}$. The doses were given either to fasting subjects or immediately after eating a high protein breakfast. The third set was the experimental measurements of Takahashi et. al. [25] of the plasma propranolol concentration after oral administration of $60 \mathrm{mg}$ of either conventional or long acting propranolol (Inderal LA) to 10 healthy subjects ( 4 females, 6 males, average weight of $59.1 \mathrm{Kg}$ ).

The mean plasma concentrations at each time point were used for the PBPK fitting. The experimental data points were obtained by using UN-SCAN-IT (Silk Scientific Corporation) to read the data from the published figures.

\section{Results}

\section{Summary of features}

PKQuest has a number of novel features, many of which have never before been implemented in a PBPK.

1) There is a large set of default parameters (e.g. organ volumes, blood flows, volume of distribution) that can be called upon. For example, all the values required for human studies are setup simply by a call to "standardhu- man". (The current version also implements a "standardrat"). The default case assumes that the solute is lipid soluble, distributes in all the body water, has a very high capillary permeability (is flow limited) and is not volatile (no pulmonary exchange). Each of these assumptions can be easily modified. For example, if the drug distributes only in the extracellular space, the user need set only the parameter "mecf" equal to 1 and PKQuest will now use the default set of extracellular volumes for each organ [4]. Or, if the drug is not flow limited but has a significant capillary permeability limitation, the user needs only to input a clearance value for the muscle capillaries ("fclear [muscle]") and all the other organs will have their permeability set on the basis of their blood flows and reference permeability surface (PS) product values relative to muscle [4]. As a another example, if the drug is volatile so that pulmonary exchange is important, the user need only input values for the two parameters Kwair (water/air partition coefficient) and Kfwat (fat/water partition) and all the appropriate parameters necessary for a respiratory compound are activated [2]. Any or all of the default values can be easily set to arbitrary, user prescribed, values.

2) The output is routinely displayed in graphical form (in addition to the standard numerical output). No user input is required to specify the routine graphical output. All of the figures shown in this paper are taken directly from the output of PKQuest without any modifications. Experimental data can be either typed in or read from a tabular file.

3) A chosen set of parameters can be optimized to give the best fit to the experimental data. PKQuest uses a rigorous non-linear global (simulated anealling $[26,27]$ ) and local (Powell, [28]) minimization routines that have a simple interface. For example, if the user wanted to find the best set of values for the Vmax and Km for the liver metabolism, and for the muscle blood flow ( "F"), it is only necessary to input "adjustn" = 3; and "adjustvar" = ["Vm[liver]", "Km[liver]", "F[muscle]"]. Any of the parameters used in the model can be optimized using this type of call and there is no limit to the number that can be simultaneously optimized (although, in most cases, simultaneous optimization of more than 3 parameters is not recommended, given the limited experimental data).

4) The rate of intestinal absorption (PO, defined as the rate of uptake by the portal vein) of a drug can be determined from the experimental venous blood levels after an oral dose. A new technique is used to invert the model equations in order to obtain an accurate solution for the PO absorption. This is a useful new application of PBPK, providing the user with an accurate time course (and graphical output) of the oral absorption rate (or, optionally, intramuscular, intraperotineal, or skin absorption) 
for any drug whose PBPK parameters have been previously determined. This is probably the single most important original feature in PKQuest, opening up a new approach to a number of pharmacokinetic problems. An example of the potential of this tool is illustrated in the application to propranolol discussed below.

5) The first pass liver metabolism (FPM) and systemic (peripheral) availability of a PO administered drug is directly determined and plotted as output. (Systemic availability is defined as the equivalent IV input that would produce an identical venous concentration as a function of time as the PO input). This determination uses a new definition of FPM that is applicable to the case of non-linear (saturable) liver metabolism. As has been discussed previously [29], the classical definition of FPM breaks down for the non-linear case and this is the first rigorous approach to describing this case. This aspect of PKQuest is presented in more detail in an accompanying paper that focuses on ethanol pharmacokinetics [3].

6) Plasma and extracellular protein binding is treated rigorously. Since the majority of drugs have some protein binding, an accurate modeling of this property is essential. The option of non-linear (saturable) protein binding is also included. Because this saturation greatly complicates the mathematics of the model, it is rarely included in PBPKs. PKQuest also allows for a combination of capillary permeability limited exchange and non-linear binding - the first time this case has been described and implemented. These features are described in more detail in an accompanying paper that discusses the application to antibiotics [4].

7) Although it is well recognized that capillary permeability may limit the blood-tissue exchange of a drug, most PBPK models deal only with the high capillary permeability (flow limited) case. PKQuest provides for a rigorous modeling of capillary permeability limitations. It is shown in an accompanying paper [4] that solutes that are normally assumed to be flow limited can have a significant capillary permeability limitation if there is a high degree of plasma protein binding.

8) The model of the lung used in PKQuest for the respiratory gases allows for the option of ventilation-perfusion mismatch. The lung can be divided into an arbitrary number of compartments (determined by the parameter N1dim) with a log normal distribution of flows and ventilation to each compartment determined by the either the default or user input values of the log standard deviation of ventilation (sig_v) and perfusion (sig_f). This feature is described in more detail in an accompanying paper that analyses the pharmacokinetics of volatile solutes [2].
9) PKQuest can be used to describe an arbitrary number of simultaneously interacting solutes. Two types of interaction (competitive or non-competitive inhibition) are allowed. Also, the case where some solutes are converted into other solutes can be modeled, with a separate Vm and $\mathrm{Km}$ for each substrate/product pair.

\section{PKQuest parameters for propranolol intravenous (IV) in- put}

Some of these features will be illustrated by application of PKQuest to propranolol pharmacokinetics. A baseline set of physiological PBPK parameters will be determined using the IV data of Fagen et. al. [24]. This data was chosen because it represents one of the most complete sets of propranolol data, with plasma values ranging from 5 minutes to 8 hours. Shown below is the listing for the procedure "prop_iv_fagen", which inputs all of the unique parameters that are required. These values have been adjusted to provide a good fit to the Fagen et. al. data.

$$
\begin{aligned}
& \text { prop_iv_fagen:=proc() } \\
& \text { defaultpar(): } \\
& \text { Wtot:=82.0; \# Total body weight } \\
& \text { standardhuman(Wtot); } \\
& \text { Fat:=0.20; Fat fraction } \\
& \text { cunit:="micrograms"; } \\
& \text { concunit[vein] :=4; } \# \text { input is plasma venous concen- } \\
& \text { tratin } \\
& \text { rblpl:=0.85; \# blood/plasma concentration ratio } \\
& \text { freepl:=0.08; \#raction free in plasma } \\
& \text { for i from } 2 \text { to } \mathrm{N} \text { do } \\
& \text { ktiss[i]:= 9.67; \#tissue/blood partition coefficients }
\end{aligned}
$$

end do; for lung

ktiss[lung]:=29.0; \# tissue/blood partition coefficient for fat

ktiss[lipid]:=2.42; \# tissue/blood partition coefficient

ktiss[muscle]:=3.62; \# tissue/blood partition coefficient for muscle

Tclr[liver]:=52; \# metabolic liver clearance 
datafile:="C:/Documents and Settings/DLevitt/My

Documents/mymaple/scandatda/ prop_iv_0_480_new"; pdata:=1; data[1]:=readtab(datafile);

\section{ninput:=1}

finput[1]:=table $([$ organ $=$ vein, type $=1$, rate $=1000$, tbeg $=0$, tend $=4.1$, csteady $=0$,

pad just=0]);\#constant infusion of 1000 micrograms/min for $4.1 \mathrm{~min}$

end proc;

The first line in the procedure ("defaultpar()") calls another procedure that sets up all the default parameters. The second line (Wtot) is the total body weight. It is the only parameter that must be set before the call to "standardhuman". (The weight is arbitrary for this data because the dose was scaled for body weight. The value of $82 \mathrm{Kg}$ was chosen because it is the average value for the Olanoff et. al. data, see below). The forth line is the fraction of body weight that is fat. The fifth line ("cunit") sets the amount unit that is used to micrograms. In PKQuest, the units of volume, weight and time are always liters, Kg and minutes. Thus, for this case, the concentration units are micrograms/liter. The parameter cunit is not needed for the actual calculations, but is used to label the printed output data and the axis of the output plot. Line 6 ("concunit") determines the units that are used for the experimental input value. In this case, concunit[vein] $=4$ indicates that the experimental input data corresponds to the venous plasma concentration. Line 7 ("rblpl") is the blood/plasma concentration ratio. If this is not entered then a default value will be used. However, in this case the experimental value is known [7].

The next 7 lines (lines 8-14) are related to the lipid binding of propranolol. Propranolol blood and tissue binding has been studied in great detail [7,30-32]. The propranolol tissue distribution seems to be determined primarily by the phosphatidylserine composition of the tissue [33]. These studies indicate that the factional binding is constant, and does not saturate over the pharmacological concentration range. Since drug binding is such a ubiquitous property of kinetic studies, PKQuest has incorporated a large number of different ways that the data can be entered. In this case, 2 sets of data are required: freepl, which is the fraction free in plasma and is set in line 8 to the experimental value [7] and ktiss[i], the ratio of the equilibrium tissue/plasma concentration which is set in lines 9-14. Lines 9-11 set ktiss $=9.67$ for all the organs, and lines 12-14 reset the ktiss for 3 organs that are known to have distinct binding (lung, muscle and lipid). Tissue binding studies indicate that propranolol binding in the lung is about 3 times higher, and muscle is about a third of that of most tissues. Somewhat surprisingly, propranolol binding to fat tissue is less than the rest of the body as indicated by a smaller volume of distribution (when normalized for body weight) in obese people [34]. Although these values of ktiss[i] are similar to the experimental measurements in the rat [33], they have been slightly adjusted to improve the fit.

It is well established that the liver is the major site of propranolol clearance. Line 15 (Tclr [liver]) is the input data describing the liver metabolism. Although it has been shown that the liver metabolism is non-linear, saturating at high blood levels, this non-linearity only becomes significant at doses above $200 \mathrm{mg} /$ day [35]. At lower doses, both the liver metabolism and PO absorption appear to be linear $[36,37]$. All the data that will be used here fall in this linear dose range. In PKQuest, there are 2 options for entering the metabolism of each organ i. If metabolism is non-linear, than the 2 parameters $\operatorname{Vmax}[\mathrm{i}]$ and $\mathrm{Km}[\mathrm{i}]$ are required and the rate of metabolism of organ $i$ is described by $\operatorname{Vmax}[\mathrm{i}]{ }^{*} c[\mathrm{i}] /(\mathrm{Km}[\mathrm{i}]+\mathrm{c}[\mathrm{i}])$ where $\mathrm{c}[\mathrm{i}]$ is the free concentration in the water of organ $i$. If the metabolism is linear, than metabolism is described by the parameter Tclr $[\mathrm{i}]$ (rate of metabolism $=\operatorname{Tclr}[\mathrm{i}]{ }^{*} \mathrm{c}[\mathrm{i}]$ ). Since the propranolol liver metabolism is linear, it is described by inputting a value of Tclr [liver] of 52 liters/min. This value was determined by using the Powel minimization feature in PKQuest to optimize the fit to the experimental data. Tclr is the "intrinsic" clearance (liters/min) of the liver, relating the rate of metabolism to the free water liver concentration. This intrinsic clearance differs significantly from the actual liver clearance (volume of plasma cleared in one pass through the liver) because the liver clearance is partially flow limited and the free water concentration is lower than the absolute concentration. Although there is some stereoselectivity in the propranolol metabolism, the effect is small and was neglected in this analysis $[38,39]$.

Line 16 ("datafile") is the name of the computer tabular file where the data (time, concentration) is stored. In line 17 , "pdata" is the number of input files, and the data is read into data $[\mathrm{i}=1]$.

The last 2 lines specify the experimental input functions. The parameter "ninput" is the number of input functions. In this case there is only one - described by the Maple table "finput[1]". The table parameter "organ" is the compartment that the input entered - in this case (IV input) the venous compartment. The parameter "type" specifies the mathematical form of the input $(0=$ bolus, $1=$ constant infusion, 2 = exponential, $3=$ Hill type). In this case there was a constant infusion at a rate of 1000 micro- 


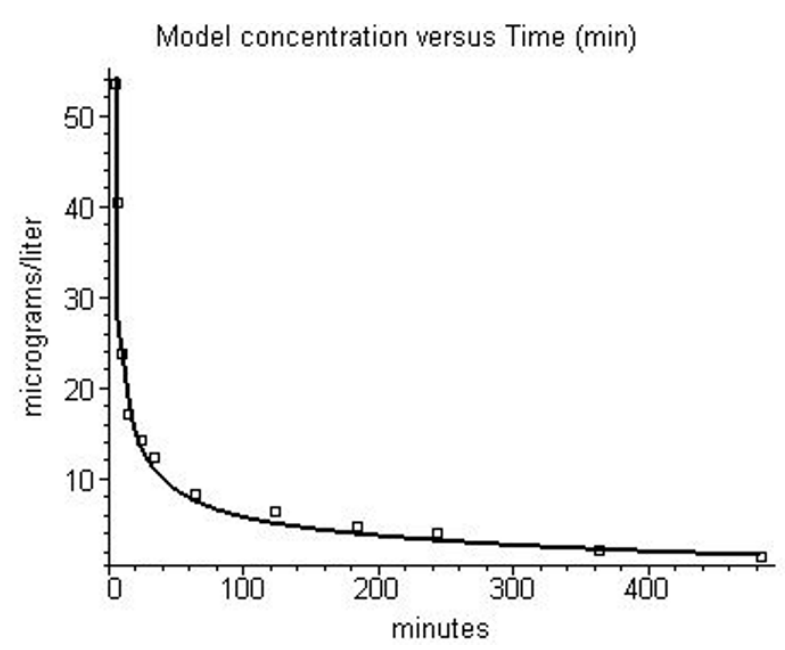

Figure 2

Comparison of PKQuest model prediction and experimental plasma propranolol concentration data (squares) of fagen et. al. [24].

grams $/$ min starting at tbeg $=0$ and ending at tend $=4.1$ minutes. (See below for an example of multiple inputs.)

This completes the description of the data entry for propranolol. There are really only 2 sets of values that are specific for propranolol: the lipid binding parameters (freepl and ktiss[i]) and the liver clearance (Tclr [liver]). All the rest of the entries (body weight, concentration units, data file, etc.) are essentially housekeeping. The default parameters (set up by the call to "defaultpar") are for the case that the solute is flow limited and distributes in all the body water. Since this is the case for propranolol, the parameters describing the volume of distribution and capillary permeability do not need to be input.

\section{PKQuest output for this set of model parameters}

Using this propranolol data, PKQuest is ready to be run. A listing of the procedure "pkquest" is shown below:

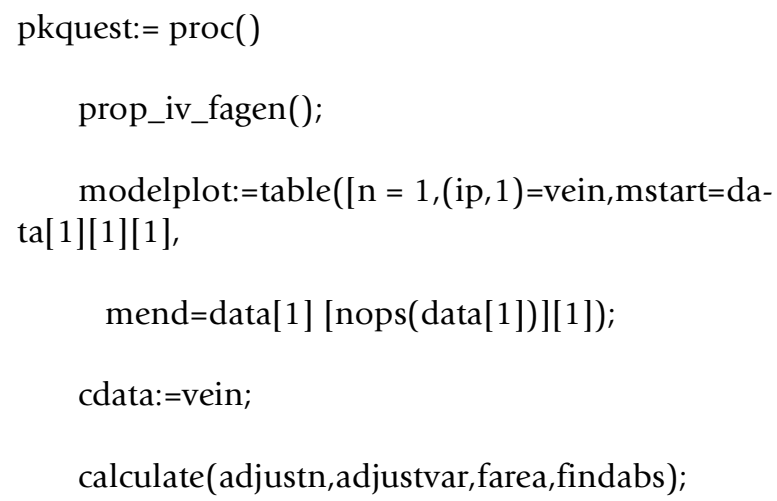

end proc;
The first line of the procedure calls the data listed above in the procedure "prop_iv_fagen". The second line calls for the standard graphical output. In this case, PKQuest will output the plot of just the venous concentration $(n=1$, (ip,1)=vein) starting at the time of the first experimental data point (data[1][1][1]) and ending at the time of the last data point (data[1] [nops(data[1])][1]). (Up to 6 different organ concentrations can be plotted on the same graph). The third line ("cdata") specifies that the venous concentration at the experimental data time points will be printed in addition to the graphical output. The last line (calculate) runs the program.

The PKQuest graphical output is shown in fig. 2. (The entire run took less than one second on a $1 \mathrm{Ghz} \mathrm{PC}$ ). The solid line is the model plasma concentration and the open squares are the experimental data. The complete layout of this figure (axes, labels, etc.) is determined without any user input. Maple has a variety of export options. The figures shown here have been exported in jpg format.

PKQuest also prints out information pertinent to each particular case, such as, in this case, the rate of liver metabolism, portal and hepatic blood flow, plasma and tissue binding, etc. PKQuest also routinely outputs the classical pharmacokinetic values for the experimental data:

Classical Phamacokinetics from Experimental Data (extrapolate to infinity): AUC $=3003.4840$ AUMC $=$ $557363.9367 \operatorname{MIT}(\min )=2.05$ clearance $($ lit $)=1.3651$ Steady State Vol. Dist. (lit) $=252.6390$

where the total clearance and steady state volume are determined from the AUC and AUMC (the area under the curve and its first moment), and MIT (the mean input time) [40].

\section{Application of PKQuest to simultaneous PO and IV input data}

Olanoff et al. [5] simultaneously administered unlabeled propranolol intravenously (IV), and deuterium labeled propranolol orally (PO) and measured the plasma levels of the labeled and unlabeled drug. Since the propranolol pharmacokinetics are linear for this dose, these two inputs can be treated as completely independent and non-interacting. These experiments will be used to quantitate the influence of food on propranolol plasma levels.

The approach that will be used is based on finding a set of PBPK parameters that are consistent with the simultaneous PO and IV input data. The model parameters must meet the following three criteria: 1) Since the measurements are simultaneous, an identical set of parameters must be used in the modeling of the IV and oral input data. 2) The PKQuest plasma levels for the IV input must fit 

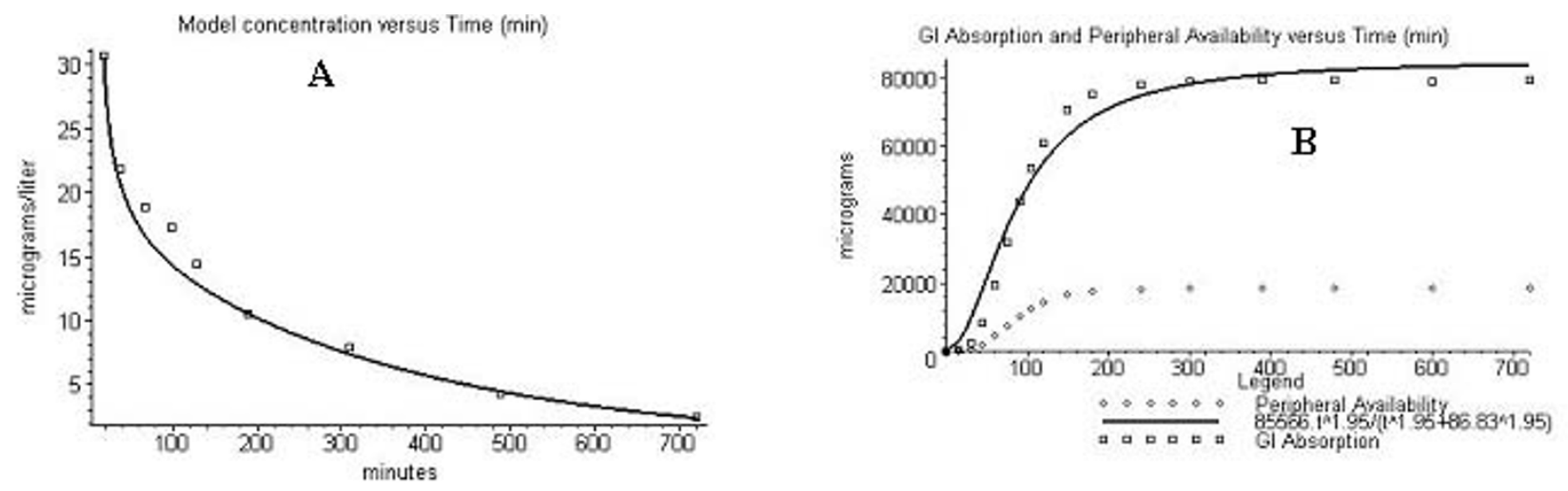

Figure 3

Comparison of PKQuest model prediction and experimental data for fasted subjects. Figure 3A shows the predicted venous plasma concentration for IV propranolol, and fig. 3B shows the predicted time course of absorption (squares) and peripheral availability (diamonds) for oral propranolol using experimental data of Olanoff et. al [5].

the observed plasma values. 3) The PKQuest total absorption for the oral input plasma data must be equal to the total experimental oral dose of $80 \mathrm{mg}$. These are quite restrictive conditions. This process will be carried out for both the fasted (fig. 3) and meal case (fig. 4) in order to determine the effect of the meal on the propranolol pharmacokinetics.

Figure 3 shows the PKQuest output for the case where the propranolol was given to fasted subjects simultaneously by the IV (fig. 3A) and PO (fig. 3B) route. Figure 3A shows a comparison of the experimental plasma data and model predictions when the propranolol was given IV $(0.1 \mathrm{mg} /$ $\mathrm{Kg}$ ). Figure $3 \mathrm{~B}$ shows the PKQuest calculation of the time course of the intestinal absorption (open squares) determined using the experimental plasma values for the oral propranolol administration. Note that, as required, the predicted total absorption equals $80 \mathrm{mg}$, the experimental oral dose. The same set of parameters is used for both figs. $3 \mathrm{~A}$ and $3 \mathrm{~B}$. All of the parameters are identical to those used above (fig. 2) for the Fagen et. al. [24] data except that it was necessary to reduce the portal blood flow by $25 \%$ from the default value used in fig. 2 in order to satisfy the three criteria stated above. This strong dependence on portal flow arises from the fact that the liver clearance is limited by liver blood flow for an IV dose, while the first pass clearance of an oral dose is relatively independent of liver flow. This relationship can be quantitated and Kornhauser et. al. [7] used measurements of the AUC after an IV and oral dose to estimate the total liver blood flow. In addition, the muscle blood flow was doubled for the fit in fig. 3 in order to slightly improve the fit. It is not clear whether this change in muscle flow has some physiological significance or is basically a sort of "fudge factor".

For the output shown in fig. 3B, the parameter "findabs" was set $=1$. This tells PKQuest to use the input blood lev- els to determine the intestinal absorption rate (see Appendix for details). (Setting findabs $=2$ will find the rate of input into the venous compartment, corresponding to IM, IP or skin absorption). Figure $3 \mathrm{~B}$ shows the standard, default PKQuest output format for this option. The open squares are the total predicted absorption as a function of time. The solid line is a 3-parameter approximation to the actual model total absorption (absorption at time $\mathrm{t}=\mathrm{A}$ $\left[\mathrm{t}^{\mathrm{H}} /\left(\mathrm{t}^{\mathrm{H}}+\mathrm{T}^{\mathrm{H}}\right)\right]$ where $\mathrm{A}=$ total absorption, $\mathrm{H}=$ Hill exponent, $\mathrm{T}=$ half time). In most cases this provides a good approximation to the PO absorption [3]. However, in this case the approximation is poor because of the unusual long initial delay in the propranolol absorption (see below for discussion). The open triangles are the total amount of systemically (peripherally) available propranolol, determined by finding the equivalent IV input that would produce the same plasma levels (see Appendix, section III, for details). The difference between the total absorption (squares) and peripheral availability (diamonds) is the first pass liver metabolism. The time dependence of the PO absorption has an initial delay of 30 to 40 minutes, followed by a relatively rapid rate of absorption that is nearly complete within about 2 hours.

A test of the accuracy of this determination of PO absorption using plasma concentration data that mimics the experimental data for propranolol is described in the Appendix, section VI. For this test case, the total predicted absorption differs from the actual total PO input by less than $1 \%$.

The next step is to repeat the above calculations, but this time using the simultaneous IV and oral data when propranolol is administered at the same time as a standard meal (660 calorie high protein breakfast [5]). As before, since the two sets of data are obtained simultaneously, exactly the same set of physiological parameters must be 

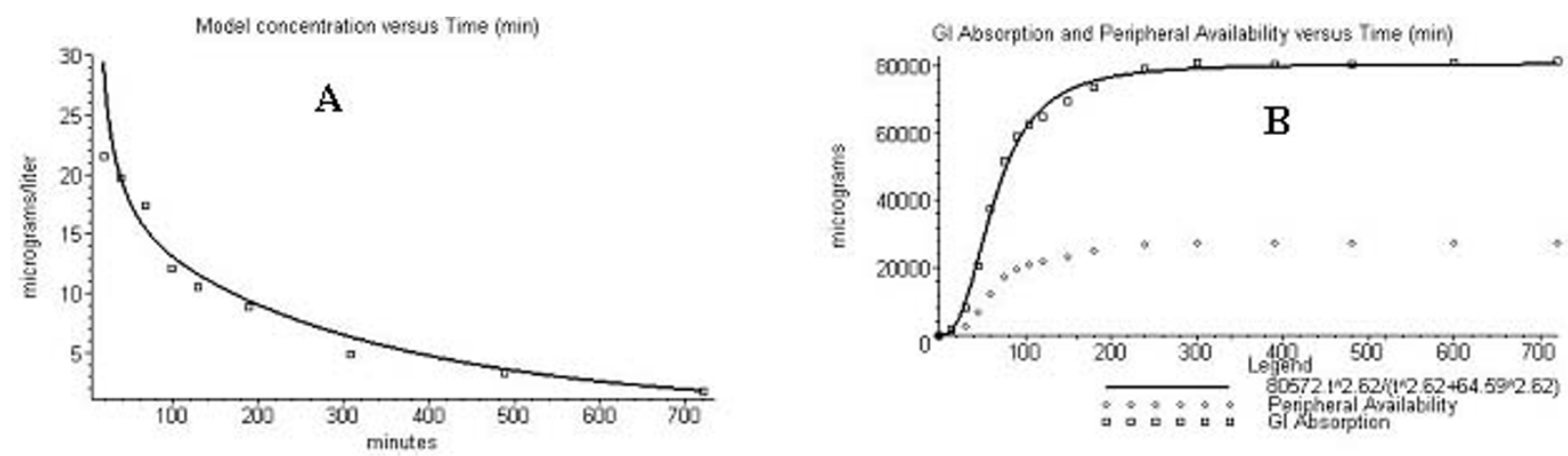

Figure 4

Same as figure 3 for case where propranolol is given either IV or orally at the same time as a standard meal.
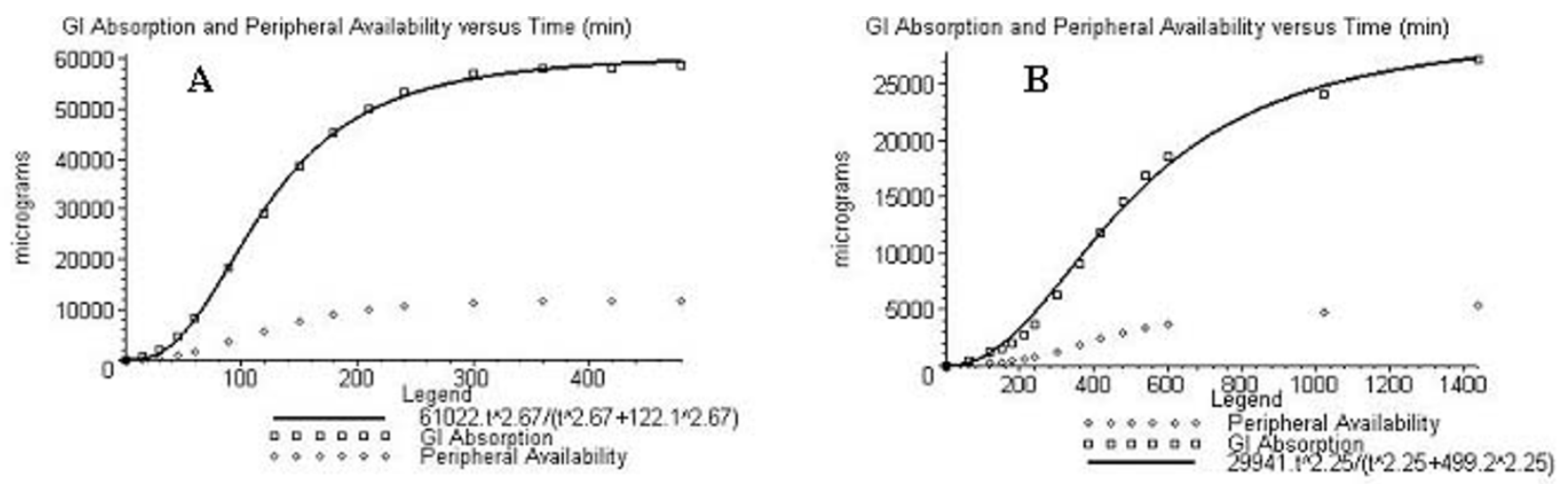

Figure 5

Time course of absorption (squares) and peripheral availability (diamonds) for oral (60 mg) standard (fig. 5A) or long acting (fig. 5B) propranolol using experimental data of Takahashi et. al [25].

used for the IV and oral calculations. Some of these parameters should differ from those used for the above calculations (fig. 3) because of the changes induced by the meal. It is well established that portal blood flow is increased during a meal. Direct measurements in humans of the time course of portal blood flow after a meal show an increase of between 50 and $100 \%$ that peaks 30 to 60 minutes after the meal [8-10]. Olfanoff et. al. [5] found an average meal induced increase in total liver blood flow of about 34\% using indocyanine green [5]. It has also been shown that the serum propranolol binding is not affected by meal induced variations in fatty acids [30] so that the model parameters related to lipid binding (freepl and ktiss[i]) were not allowed to vary between the fasting and standard meal experiments.

As described above, meals have a bi-directional effect on blood levels: decreasing the plasma concentration (and AUC) when propranolol is given IV, and increasing the levels (and AUC) when given orally. This bi-directional meal effect could not be fit just by varying the portal blood flow. In order to quantitatively fit the meal data, it was necessary to increase the value used above for the Olanoff et. al fasting portal blood flow (F [portal]) by $50 \%$ and decrease the "intrinsic" liver propranolol clearance (Tclr [liver]) by $20 \%$. The increased portal flow is consistent with the indocyanine green measurements of Olanoff et. al. [5]. Figure 4 shows the PKQuest fit to the meal data using this modified parameter set. As before, the requirements of a "successful" fit to the simultaneous data is that the IV input model curve fit the observed plasma concentration (fig. 4A) and the predicted total absorption for the PO input case (fig. 4B) equal the actual amount administered (80 mg).

\section{Application of PKQuest to a slow release form of pro- pranolol}

In this section, PKQuest will be applied to the data of Takahashi et. al [25] in which the oral absorption (in fasting subjects) of a sustained release form of propranolol (Inderal LA) was compared with the standard form in same set of subjects. The PBPK parameters used for these 
subjects were identical to those determined above for the IV data of Fagen et. al. [24], except that the value of Tclr [liver] was adjusted (increased by $50 \%$ per $\mathrm{Kg}$ body weight) so that the total absorption for the standard propranolol equaled the total oral dose $(60 \mathrm{mg})$. Figure 5 shows the predicted time course for the experimental data for oral administration of the standard form (fig. 5A) and the long acting form (fig. 5B). It can be seen that the absorption rate is markedly slowed. There appears to be a significant absorption rate even for the last data point at 24.5 hours. It can also be seen that only about $50 \%$ of the slow release form is absorbed by 24 hours $(30 \mathrm{mg}$ absorption of the $60 \mathrm{mg}$ dose). It is possible that a higher absorption faction would have been seen if the plasma measurements were carried out for longer times. This result is consistent with the absorption estimate of $60 \%$ of Takahashi et. al [25], based on measurements of the 48 hour urinary excretion of 6 metabolites of propranolol.

Once the PBPK parameters have been determined, PKQuest can be used to predict the blood levels for an arbitrary set of input functions. For example, using the above analysis, one can determine the blood levels after different dosage regimens of the standard and long acting forms of propranolol. This will be illustrated by a comparison of the blood levels of a daily dose of $120 \mathrm{mg}$ of the standard form given in $40 \mathrm{mg}$ doses, 3 times a day (at 8 hour intervals) versus $120 \mathrm{mg}$ of the sustained release form (Inderal LA) given once a day. (According to the Physicians Desk Reference, these are roughly equivalent therapeutic doses). The PO absorption rate $(\mathrm{Q}(\mathrm{t}))$ is described by the 3 parameter Hill equations obtained from the PKQuest output shown in fig. 5A (standard) and 5B (long acting) where $\mathrm{Q}(\mathrm{t})=\mathrm{A}\left[\mathrm{t}^{\mathrm{H}} /\left(\mathrm{t}^{\mathrm{H}}+\mathrm{T}^{\mathrm{H}}\right)\right]$ and $\mathrm{A}=$ total absorption, $\mathrm{H}=$ Hill exponent, $\mathrm{T}=$ half time. For the case of the 3 times per day $40 \mathrm{mg}$ standard dose: $\mathrm{A}=40000 \mu \mathrm{g}$, $\mathrm{H}=2.67$, and $\mathrm{T}=122.1$ (fig. $5 \mathrm{~A}$ ). For the case of the once per day $120 \mathrm{mg}$ long acting dose: $\mathrm{A}=60000 \mu \mathrm{g} ; \mathrm{H}=2.25$ and $\mathrm{T}=499.2$ (fig. $5 \mathrm{~B}$ ). The value of $\mathrm{A}$ (total amount absorbed) for the long acting form was assumed to be 60 $\mathrm{mg}, 50 \%$ of the total $120 \mathrm{mg}$ dose.

PKQuest has a simple interface for entering arbitrary input doses. The following input is for the case of 4 consecutive $40 \mathrm{mg}$ doses of the standard form at 8 hour intervals:

ninput:=4;

finput $[1]:=$ table $([$ organ $=$ liv-

er, type $=3$, rate $=40000$, tbeg $=0$, tend $=122.1, \mathrm{hn}=2.67$, cstead $\mathrm{y}=0$, padjust $=0]$ );

finput[2]:= table $([$ organ $=$ liv-

er, type $=3$, rate $=40000$, tbeg $=480$, tend $=122.1$, hn $=2.67$, cste ady $=0$, padjust $=0]$; finput[3]:= table $([$ organ $=$ liv-

er, type $=3$, rate $=40000$, tbeg $=960$, tend $=122.1$, hn $=2.67$, cste $\operatorname{ady}=0$, padjust $=0])$;

finput $[4]:=$ table $([$ organ $=$ liv-

er, type $=3$, rate $=40000$, tbeg $=1440$, tend $=122.1$, hn $=2.67$, cst eady $=0$, padjust $=0]$ );

The input is $\mathrm{PO}$ absorption directly to the liver (organ=liver), described by the Hill equation (type = 3) with A, H and $\mathrm{T}$ indicated by rate, tend and hn, respectively. The parameter tbeg indicates the start time in minutes of each input. (The parameters csteady and padjust are not used for this case). Figure 6 shows the predicted blood levels using the Takahashi et. al. PBPK parameters for the 3 times per day standard propranolol (fig. 6A) and the once per day long acting form (fig. 6B). (This calculation of blood levels for 32 hours required 1.7 seconds on a $1 \mathrm{Mhz}$ computer). It can be seen that the once per day $120 \mathrm{mg}$ long acting dose has significantly lower blood levels then the same daily dosage of the standard form.

\section{Discussion and Conclusions Overview of novel aspects of PKQuest}

PKQuest differs from other PBPKs in that it has been designed to be a complete general-purpose modeling program. To this end it includes all the different features that are required to model the commonly investigated compounds. In this and the accompanying papers [2-4], examples of the application of PKQuest to the human kinetics for $\mathrm{D}_{2} \mathrm{O}$, inulin, propranolol, ethanol, toluene, anesthetic gases, ceftriaxone and dicloxacillin (protein bound antibiotics) are presented. All of these studies use the same set of basic physiological parameters. (For most of these compounds, these results represent the first complete human PBPK.) These compounds cover most of the range of chemical properties that one would expect to encounter in a pharmacokinetic study. The pharmacokinetics of different solutes depends on different sets of PBPK parameters. For example, the time course of the distribution of $\mathrm{D}_{2} \mathrm{O}[22]$ has a strong dependence on the cardiac output, muscle blood flow and total body water, while the kinetics for inulin [41] have a strong dependence on the extracellular volume and capillary permeability Knowing that the same PBPK (using the same set of parameters) is applicable to a large variety of compounds increases ones confidence in the validity of the parameters.

The three accompanying papers illustrate other novel features of PKQuest: a) quantitation of first pass metabolism for non-linear liver metabolism [3]; b) measurement of capillary permeability [4]; c) description of non-linear plasma and tissue protein binding [4]; d) pharmacokinetics of volatile solutes [2]; and e) importance of perfusionventilation mismatch [2]. Each of these papers provides 

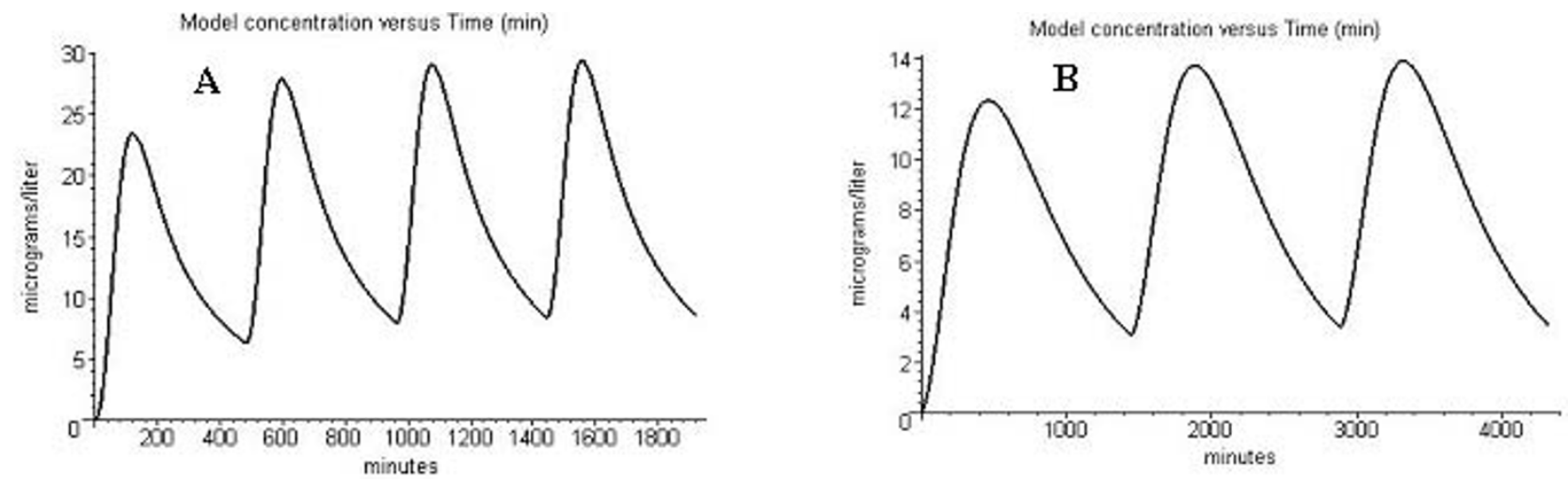

Figure 6

Simulated venous plasma concentration for oral administration of standard (fig. 6A) and sustained release (fig. 6B) form of propranolol: A) Standard form: $40 \mathrm{mg}$, three times per day; B) Sustained release form: $120 \mathrm{mg}$, once per day.

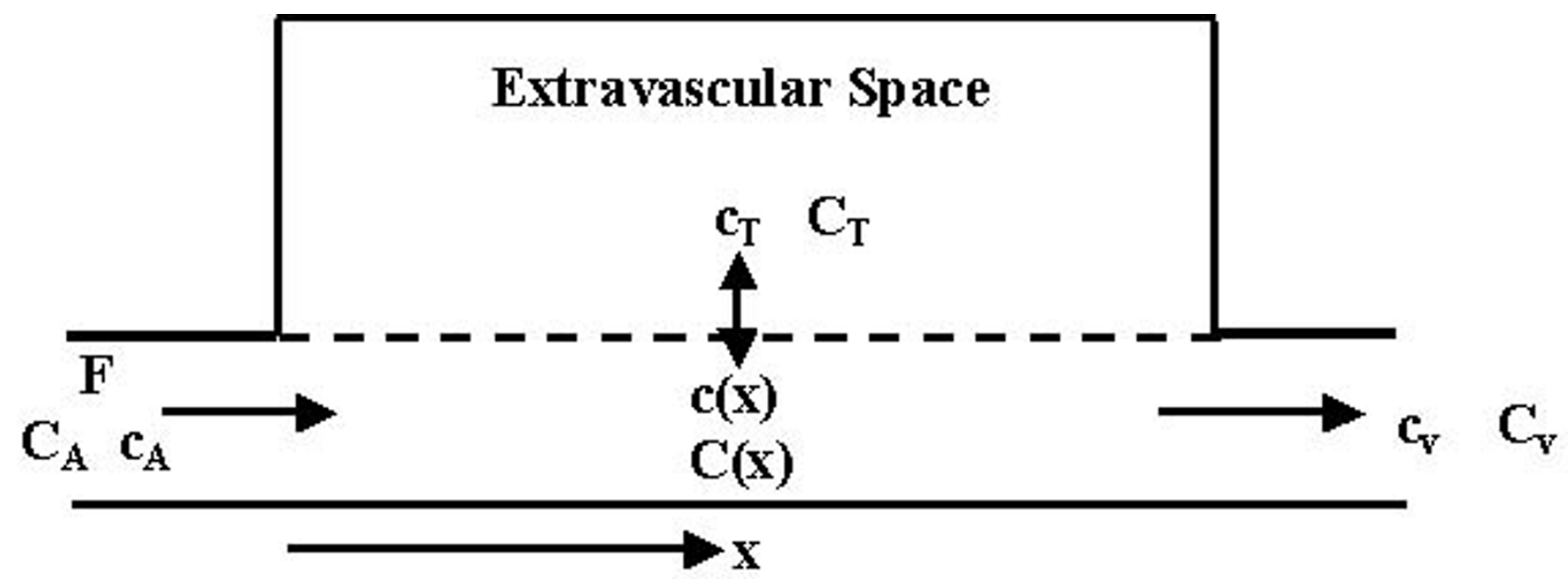

Figure 7

Diagram showing the parameters involved in the exchange of solute between the capillary and the extravascular (tissue) space. The variable $C$ is the total concentration, $c$ is the free water concentration and $F$ is the blood flow. The subscript $A$ indicates arterial, $\mathrm{T}$ tissue and $\mathrm{V}$ venous compartments.

significant new information about intensively investigated solutes.

The most important of the new features is the option that provides a quantitative measurement (and graphical output) of the time course of the intestinal absorption and peripheral (or systemic) availability. Calculations of intestinal absorption have been incorporated into earlier PBPK calculations based on the assumption that intestinal absorption could be described by either a simple first order rate process [42-44], or by a pseudo two [45] or N [46] multi-compartment process. These intestinal compartmental parameters were then adjusted to provide the optimal fit to the observed blood time course. In contrast, in
PKQuest, the PBPK equations are directly solved for the intestinal absorption time course without any assumptions about the physical or mathematical form of the absorption process.

If the system is linear, another method of estimating the intestinal absorption or peripheral availability is by the application of the deconvolution approach to the blood levels after an IV and PO input in the same subject [47]. For the peripheral availability, this technique provides a direct approach with a minimum of model assumptions. In contrast, the intestinal absorption cannot be directly determined by the deconvolution approach without some additional assumptions [47] or by using experimental 
measurements of the systemic blood levels produced by portal vein input $[48,49]$. Thus, the intestinal absorption feature of PKQuest should provide a useful new application even for the linear case.

The use of PKQuest to determine the intestinal absorption or peripheral availability requires that PBPK parameters first be determined using the IV input measurements. However, this does not mean that these measurements must be made in the same individuals (as is a requirement for the strict deconvolution approach). For example, in the above measurement of the rate of intestinal absorption of the long acting form of propranolol, no IV input information was available for these subjects. The PBPK parameters for these subjects were determined by using the previously determined parameters, inputting the body weight and fat fraction for these subjects and by using the assumption that the short acting form was completely absorbed to determine the value of the intrinsic liver clearance for these subjects.

The term "intestinal absorption" used in PKQuest refers to the total amount that enters the portal vein. Drug that is absorbed and then metabolized by the GI tract before entering the portal vein will be treated as if it was never absorbed. For the special case of a drug that is known to be completely absorbed (e.g. ethanol), the difference between the total PKQuest "absorption" and the total PO dose is a measure of the GI tract drug metabolism.

PKQuest provides the first, rigorous, operational definition of peripheral availability and first pass metabolism (FPM) for the non-linear case. As discussed in detail in the application of PKQuest to ethanol metabolism [3], confusion about the definition of FPM has been the cause of a controversy regarding the relative importance of gastric metabolism in the overall ethanol metabolism.

One of the most important applications of animal PBPKs is to predict the time course of drug levels in different tissues. This feature must be used warily when applied to humans because the tissue partition coefficients are, in most cases, not directly measured but are extrapolated from animal measurements. The successful prediction of the venous plasma concentrations (e.g. figs. 2,3,4) does not guarantee that the correct tissue partition coefficients have been used, especially in organs with small volumes (e.g. brain) that have little influence on the total body disposition. The output of PKQuest allows the simultaneous display of the concentrations in up to 6 different organs.

\section{Effect of meals on propranolol pharmacokinetics}

The application of PKQuest to the simultaneous measurements of Olanoff et. al. [5] indicates that a meal has two actions on propranolol metabolism: 1) An increase in portal blood flow; and 2) A decrease in the intrinsic liver metabolism of propranolol. This meal induced increase in portal blood flow is a well documented effect $[5,8-10]$. In contrast, the decrease in metabolism has not been previously quantitated and Olanoff et. al. [5] suggested that the increase in portal blood flow might be the only change required to explain the meal effect. The decreased intrinsic clearance presumably results from some competition in the liver between the absorbed food products and propranolol.

There is another rather peculiar effect of meals on propranolol kinetics that is not usually commented on. A close look and at the plasma blood level measurements of Melander et. al. [50] and Walle et. al. [51] reveals that the peak in the plasma concentration curve for the meal case occurs at a slightly earlier time than for the fasting case. This is the opposite of what would be expected because the meal will significantly delay the rate of gastric emptying which should delay the peak in the plasma concentration. For example, the peak in the blood ethanol after an oral dose is delayed by about 20 minutes by a meal because of the delayed gastric emptying [3,52]. The above PKQuest analysis provides an explanation for this peculiar effect. A comparison of figs. 3B and $4 \mathrm{~B}$ shows that in the fasting case (fig. 3B) there is a long delay (about $40 \mathrm{~min}$ utes) before any significant PO absorption occurs. This initial delay is significantly reduced in the meal case (fig. $4 \mathrm{~B}$ ). This 40 minute initial delay suggests that some sort of change in the state of propranolol (e.g. lipid binding or micelle formation) is required before it can be absorbed and that this change is more rapid in the presence of a meal. Whatever the explanation, this ability to obtain high resolution information about the time course of $\mathrm{PO}$ drug absorption is a useful feature of PKQuest.

In addition to providing quantitative information about the rate of $\mathrm{PO}$ drug absorption and peripheral availability, PKQuest also routinely provides a 3 parameter equation describing the rate of $\mathrm{PO}$ absorption that can be used to predict blood levels for other dosage regimens. This was illustrated above in a comparison of the same total daily propranolol dose given either 3 times per day using standard propranolol or once per day as the long acting form. This same approach can be used to predict blood levels administered via IM, IP or skin.

\section{Competing interests}

None declared.

\section{Appendix: Details of PKQuest} I. Given a known input to the animal, solve for the venous, arterial and organ concentrations as a function of time

The derivation of the basic set of model equations is described here. This derivation takes pains to distinguish 
clearly between weights and volumes of organs and flows and between the total organ concentration and the free water concentration. In PKQuest, volume, weight and time are always in units of liters, $\mathrm{Kg}$ and minutes. The solute quantity unit (moles, gms, etc.) is arbitrary and is set by the user. For illustrative purposes, it has been assumed below that the unit is moles. The following definitions are used:

$\mathrm{v}_{\mathrm{i}}=$ weight $(\mathrm{Kg})$ of organ $\mathrm{i}$

$\mathrm{C}_{\mathrm{i}}=$ amount $/ \mathrm{Kg}(\mathrm{mole} / \mathrm{Kg})$ in organ $\mathrm{i}$.

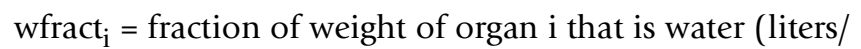
$\mathrm{Kg})$

$\mathrm{c}_{\mathrm{i}}=$ free concentration in water (moles/liter)

$\mathrm{fw}_{\mathrm{i}}=$ fraction of the total solute in organ $\mathrm{i}$ that is free $=\mathrm{c}_{\mathrm{i}^{-}}$ wfract $_{\mathrm{i}} / \mathrm{C}_{\mathrm{i}}$

ecf $f_{\mathrm{i}}=$ fraction of extravascular water that is extracellular

$F_{i}=$ organ blood flow (liters/min)

$\mathrm{PS}_{\mathrm{i}}=$ permeability - surface area product for organ i(liters/ $\min / \mathrm{Kg}$ )

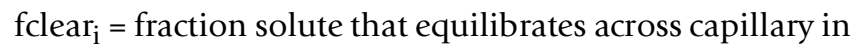
one pass

Valv = Lung alveolar gas volume per lung weigt (liters/Kg) alvent $=$ Lung alveolar ventilation rate (liters/min)

$\rho_{B}=$ density of blood (Kg/liter)

$\mathrm{k}=$ protein binding constant (moles/liter) ${ }^{-1}$

$\mathrm{Pb}_{\mathrm{i}}=$ concentration of protein binding sites in organ $\mathrm{i}$ (moles/liter) $\mathrm{Q}_{\mathrm{i}}=$ metabolism by organ $\mathrm{i}$ (moles/min)

cinput $_{\mathrm{i}}=$ external input to organ $\mathrm{i}(\mathrm{moles} / \mathrm{min})$

It will be assumed in this section that solute binding is linear and does not saturate $\left(\mathrm{fw}_{\mathrm{i}}=\mathrm{constant}\right)$. The modifications of the equations required for non-linear binding will be described in section IV. The solution described in this section uses the conventional approach that is routinely used in PBPK models. The only aspect that is novel is the incorporation of a capillary permeability limitation through the parameter fclear ${ }_{i}$.

Figure 7 describes the exchange of solute between the blood and tissue compartment across the capillary wall. It is assumed that the tissue consists of $\mathrm{N}$ capillaries/Kg, all with exactly the same geometry, blood flow, permeability, etc. It will also be assumed that the relation between the capillary and tissue concentration is in a pseudo steady state. The steady state differential equation for the concentration in the capillary as a function of position is:

$$
-\rho_{B}(F / v) \frac{d C_{B}}{d x}=2 \pi a P N\left[c_{B}(x)-c_{T}\right]
$$

where $\mathrm{C}$ and $\mathrm{c}$ are the total and free water concentration, subscript $\mathrm{B}$ and $\mathrm{T}$ refers to the blood and tissue compartment, $\mathrm{P}$ is the capillary permeability, $\mathrm{N}$ is the number of capillaries per $\mathrm{Kg}$ tissue, and a is the capillary radius. The total concentration $C_{B}$ can be related to the free concentration $\left(c_{B}\right)$ using the definition of $\mathrm{fw}_{\mathrm{b}}\left(\mathrm{C}_{\mathrm{B}}=\mathrm{c}_{\mathrm{B}} \mathrm{wfract}_{\mathrm{b}} /\right.$ $\mathrm{fw}_{\mathrm{b}}$ ). Integrating eq. 1 over the length of the capillary and solving for the venous concentration leaving the capillary:

$$
c_{v}=c_{T}+\left[c_{A}-c_{T}\right] \exp \left(-\frac{f w_{B} P S}{\text { fract }_{B} \rho_{B}(F / v)}\right)
$$

where PS is the permeability-surface area product per tissue weight (liters/min/Kg). Equation 2 can then be related to capillary clearance term fclear[i] (for organ i) that is used in PKQuest:

$$
\text { fclear }[i]=\left(c_{A}-c_{V}\right) /\left(c_{A}-c_{T}\right)=1-\exp \left(-\frac{f w_{B} P S_{i}}{w \operatorname{fract}_{B} \rho_{B}\left(F_{i} / v_{i}\right)}\right)
$$

where the dimensionless fclear is equal to the fractional equilibration that occurs in one pass through the capillary. Note that physical capillary permeability (PS) in eq. 3 is multiplied by $\mathrm{fw}_{\mathrm{b}}$, the fraction free in the blood. Thus, solutes that have a high intrinsic permeability may become permeability limited if a large fraction is bound. This case is discussed in detail in the application of PKQuest to protein bound antibiotics [4].

Using eq. 3 to relate the venous concentration $\left(c_{V}\right)$ to the tissue concentration $\left(c_{T}\right)$ and the definition of fw to relate the free to total concentration, the differential equation for the free tissue concentration in organ $i\left(c_{i}\right)$ can be obtained from the mass balance relation for organ $\mathrm{i}$ :

$$
v_{i} \frac{w \text { ract }_{i}}{f w_{i}} \frac{d c_{i}}{d t}=\rho_{B} F_{i} \frac{w \text { ract }_{B}}{f w_{B}} \text { fclear }_{i}\left(c_{B}-c_{i}\right)+\text { cinput }_{i}-Q_{i}
$$

where $\mathrm{Q}$ is the metabolic utilization and cinput is the input from outside the body. In equation $4, \mathrm{C}_{\mathrm{B}}$ is the free concentration entering the organ. For the lung $\mathrm{C}_{\mathrm{B}}$ corresponds to the venous concentration and for all other organs $c_{B}$ is the arterial concentration. If the solute can only distribute in the extracellular space, than the wfract $_{i}$ term in eq. 4 is replaced by the product ecf $f_{i} \times$ wfract $_{i}$ where ecf $f_{i}$ is the fraction of extravascular water in organ $i$ that is extracellular. 
The mass balance equation for the lung must be modified to take account of ventilatory exchange of volatile solutes. The total concentration of solute in the lung $\left(\mathrm{C}_{\mathrm{L}}\right)$ is the sum of the solute in the lung tissue compartment plus that in the alveolar gas space:

$$
C_{\mathrm{L}}=\operatorname{ValvC}_{\mathrm{Alv}}+C_{\mathrm{T}}=\operatorname{Valv}_{\mathrm{Alv}}+\text { wract }_{\mathrm{L}} \mathcal{C}_{\mathrm{L}} / f w_{\mathrm{L}}
$$

where Valv is the alveolar volume per unit weight (liters/ $\mathrm{Kg}$ ) and $\mathrm{C}_{\mathrm{T}}$ is the absolute tissue concentration which can be related to the free concentration in lung water $\left(c_{L}\right)$ via $\mathrm{fw}_{\mathrm{L}}$. It is assumed that the solute in alveolar gas is in equilibrium with the lung tissue water:

$$
C_{\mathrm{Alv}}=c_{\mathrm{L}} / \text { Kwair }
$$

where Kwair is the water/air partition coefficient. Thus:

$$
C_{L}=\left(\text { Valv / Kwair }+ \text { wract }_{L} / f w_{L}\right) c_{L}=\text { wract }_{L} c_{L} / f w_{L}^{\prime}
$$

$$
f w_{L}^{\prime}=\frac{w \text { fract }_{L}}{\text { Valv } / \text { Kwair }+w \text { fract }_{L} / f w_{L}}
$$

Thus, eq. 4 can also be used for the lung, with fw' substituted for fw. The cinput term in eq. 4 for the lung can be written in terms of the alveolar ventilation (alvent, liters/ minute):

$$
\operatorname{cinput}_{\mathrm{L}}=\operatorname{alvent}\left(C_{\text {inhale }}-c_{\mathrm{L}} / \text { Kwair }\right)
$$

where $\mathrm{C}_{\text {inhale }}$ is the inhaled gas concentration (moles/liter). The above equations are for the case where there is one lung compartment. The modifications for the case of perfusion-ventilation mismatch is described in section $\mathrm{V}$.

Equation 4 is the mass balance for each organ compartment. We still need to define the mass balance for the arterial and venous compartments. For the artery, the mass balance is described by:

$$
v_{A} \frac{w \text { fract }_{B}}{f w_{B}} \frac{d c_{A}}{d t}=\rho_{B} F_{C O} \frac{w \text { fract }_{B}}{f w_{B}}\left(c_{V}^{L}-c_{A}\right)+\operatorname{cinput}_{A}-Q_{A}
$$

where the subscript $\mathrm{A}$ indicates artery, $\mathrm{F}_{\mathrm{CO}}$ is the cardiac output and $\mathrm{C}_{\mathrm{v}}^{\mathrm{L}}$ is the concentration in the venous blood leaving the lung. The concentration $\mathrm{c}_{\mathrm{v}}^{\mathrm{L}}$ can be related to the venous concentration entering the lung $\left(c_{V}\right)$ by the use of eq.3:

$$
c_{V}^{L}=c_{V}-\text { fclear }_{L}\left(c_{V}-c_{L}\right)
$$

Finally, substituting eq. 10 into eq. 9:

$$
v_{A} \frac{w \text { fract }_{B}}{f w_{B}} \frac{d c_{A}}{d t}=\rho_{B} F_{C O} \frac{\text { fract }_{B}}{f w_{B}}\left[\left(1-\text { fclear }_{L}\right) c_{V}+\text { fclear }_{L} c_{L}-c_{A}\right]+\operatorname{cinput}_{A}-Q_{A}
$$

An equation similar to eq. 9 is obtained for the venous compartment, except that it is necessary to sum over the venous concentrations leaving each organ, multiplied by the organ blood flow:

$$
v_{V} \frac{w \mathrm{fract}_{B}}{f w_{B}} \frac{d c_{V}}{d t}=\rho_{B} \frac{w \mathrm{fract}_{B}}{f w_{B}}\left[\sum_{i=2}^{11} F_{i} c_{V}^{i}-F_{C O} c_{V}\right]+\operatorname{cinput}_{V}-Q_{V}
$$

where $\mathrm{c}_{\mathrm{V}}^{\mathrm{i}}$ is the concentration in the vein leaving organ $\mathrm{i}$. The sum is over all the organs that empty in to the vein ( $\mathrm{i}$ $=0$ and 1 correspond to the venous and arterial compartments). The concentration $\mathrm{c}_{\mathrm{V}}$ can be related to the arterial concentration entering organ i by the use of eq.3:

$$
c_{V}^{i}=c_{A}-\text { fclear }_{i}\left(c_{A}-c_{i}\right)
$$

Finally, substituting eq. 13 into eq. 12:

$$
\text { (14) } v_{V} \frac{w \text { fract }_{B}}{f w_{B}} \frac{d c_{V}}{d t}=\rho_{B} \frac{w f r a c t}{f w_{B}}\left[\sum_{i=2}^{11} F_{i}\left(c_{A}-\text { fclear }_{i}\left(c_{A}-c_{i}\right)\right)-F_{C O} c_{V}\right]+\text { cinput }_{V}-Q_{V}
$$

The set of differential equations for $\mathrm{dc}_{\mathrm{i}} / \mathrm{dt}, \mathrm{i}=0 . .11$ for each of the organs $(i=2 . .11)$ and the venous $(i=0)$ and arterial $(\mathrm{i}=1)$ compartments is then solved by a RungeKutta numerical procedure for the values of $c_{i}$ as a function of time. The free water concentration $c_{i}$ is then converted to the experimental concentration units and plotted along with the experimental data. This conversion depends on exactly what is measured experimentally. In PKQuest the user inputs the parameter concunit[i] which characterizes what experimental unit is used. For example, concunit $=1$ indicates total concentration in units of amount $/ \mathrm{Kg}$ wet weight; concunit $=2$ corresponds to amount/liter tissue; concunit $=4$ corresponds to plasma concentration, etc.

\section{Given the experimental venous concentration as a func- tion of time, solve for rate of oral (PO) or intramuscular (IM) absorption as a function of time}

The solution described above in I corresponds to the case where the input to one or more of the compartments is given (cinput[i]) and the concentration of the different compartments as a function of time is solved for. This section describes the new procedure that was developed to invert this solution, and find the value of cinput[i] as a function of time that would produce the experimental venous concentration. Although the approach described below can be used for input into any compartment, the current version of PKQuest implements only two different inputs: 1) PO input in which the solute is absorbed from the GI tract and goes directly into the portal vein, where it distributes in and is metabolized by the liver before entering the systemic vein; and 2) IV input in which the solute directly enters the systemic vein. The IV input case is applicable to any input where there is time dependent input directly to the venous blood without significant distribu- 
tion in another organ - e.g. intramuscular (IM), intraperitoneal (IP) or skin absorption.

Since the venous concentration is known experimentally at a set of time points, this concentration can be used as input to the set of equations described in section I (omitting the venous differential equation, since its concentration is known). The solution to this set of equations then yields the corresponding arterial and organ concentrations as a function of time. In the implementation in PKQuest, a cubic spline interpolation is applied to the experimental data points to obtain a continuous function $c_{v}(t)$ that is used as input to the set of equations. For the case of IV input these organ concentrations (as a function of time) should be equal to the actual organ concentrations in the experimental case, since both cases have the same venous concentration.

For the PO input case, all the organs will have the correct concentration except for the liver which receives input from the GI tract in addition to the venous input. Another step is required to determine the liver concentration for this case. The differential equation for the venous compartment (eq. 14) can be solved for the liver concentration $\left(\mathrm{c}_{\text {Liver }}(\mathrm{t})\right)$ since the concentrations in all the other organs and in the vein are known. (In eq. 14, the cinput term is 0 since there is no venous input, and $\mathrm{Q}_{\mathrm{V}}$ is zero because it is assumed for this case that there is no metabolism in the venous compartment). The only factor in eq. 14 that is not directly available is the time derivative of the venous concentration $\left(\mathrm{dc}_{\mathrm{v}} / \mathrm{dt}\right)$. However, this can be estimated by taking the derivative of the cubic spline interpolation to the experimental venous data. This solution for the liver concentration is then combined with the previous solutions to obtain the concentrations of all the body organs as a function of time for the PO input case.

Knowledge of the concentrations as a function of time of all the body compartments is all that is needed to determine the rate of input to the body. The total body input at time $\mathrm{t}$ is equal to the total amount in the body at time $\mathrm{t}$ (obtained by summing over all the body compartments) plus the total amount that has been metabolized or excreted up to that time - which is obtained by integrating over the rates of metabolism $\left(\mathrm{Q}_{\mathrm{i}}\right)$ in the different organs, plus the rate of renal clearance, etc. In the implementation of PKQuest, this calculated total amount input is plotted as a function of time. A test of this approach is described below (VI) using data that is representative of what is observed experimentally for propranolol. It is shown that the total predicted absorption differs from the actual total PO input by only about $0.5 \%$.

\section{Direct calculation of the first pass metabolism or pe- ripheral availability for the case of $P O$ input}

The procedure described above in section II for determining the rate of $\mathrm{PO}$ absorption can be directly used to determine the first pass metabolism. In the calculation in section II, two different sets of liver concentrations were obtained: Case 1) Using just the experimental venous concentration as input. Case 2) Using the direct calculation of the liver concentration via the solution of eq. 14. Case 1 corresponds to the liver (and other organ) concentrations that would be obtained if the input were intravenous (IV) and Case 2 is for a PO input. Both cases have exactly the same venous concentration. This means that both cases have the same "peripheral availability". Thus, the peripheral availability for the PO input case can be obtained by solving for the IV input as a function of time (by summing over the amounts in each organ plus the amount of metabolism) using the organ concentrations for Case 1. This is a direct measure of what the time course of the IV input would have to be to give exactly the same venous concentrations as occurred for the PO input. The first pass metabolism (FPM) is directly related to the peripheral availability by the following relation:

First Pass Metabolism $=$ Total Absorption - Peripheral Availability

In the implementation of PKQuest for the PO absorption case, both the total amount of PO absorption and the peripheral availability are routine plotted as a function of time.

This is a new definition of first pass metabolism. It provides the first rigorous, operational definition of FPM that is applicable to the non-linear liver metabolism case. This case is discussed in more detail in the paper describing the application of PKQuest to ethanol pharmacokinetics [3].

\section{Modifications of above equations for the case where there is non-linear (saturable) protein binding}

Allowing for non-linear protein binding seriously complicates the model. It corresponds to the case where the $\mathrm{fw}_{\mathrm{i}}$ in the above equations becomes a function of $\mathrm{c}_{\mathrm{i}}(\mathrm{t})$ and it requires major modifications of all of the equations. The modifications described below are similar to those used previously by Tsuji, et. al. [53], but with the new feature of including the capillary permeability. This is the first description of a PBPK model that allows for both non-linear binding and capillary permeability.

The modifications of the left and right hand sides of eq. 4 will be described separately. The left hand side described the change in the total (bound + free) concentration in organ i: 


$$
v_{i} \frac{d C_{i}}{d t}=v_{i} w \text { fract }_{i}\left[\frac{d c_{i}^{b}}{d t}+\frac{d c_{i}}{d t}\right]
$$

where, as previously defined, $c_{i}$ is the free water concentration, and $c^{b}{ }_{i}$ is the concentration of the bound solute in the organ water. It will be assumed that there is only one homogeneous binding site with the classical Scatchard type binding described by:

$$
\text { fractionfree }=f w_{i}=\frac{1+k c_{i}}{1+k c_{i}+k P b_{i}}
$$

$$
c_{i}^{b}=\frac{1-f w_{i}}{f w_{i}} c_{i}=\frac{k P b_{i} c_{i}}{1+k c_{i}}
$$

where $\mathrm{k}$ is the binding constant for the protein and $\mathrm{Pb}_{\mathrm{i}}$ is the concentration of protein binding sites in compartment i. Pb must have the came units as c (e.g. moles/liters) and $\mathrm{k}$ has the inverse units (e.g. $1 /$ (moles/liters)). It is assumed that there is only one type of binding protein (with a unique $\mathrm{k}$ ) that is distributed with different concentrations $\left(\mathrm{Pb}_{\mathrm{i}}\right)$ in the different compartments. Taking the derivative of $c^{b}{ }_{i}$ (eq. 16) and substituting it in eq. 15:

$$
\begin{aligned}
& \frac{d c_{i}^{b}}{d t}=\frac{k P b_{i}\left(1+k c_{i}\right)-k^{2} P b_{i} c_{i}}{\left(1+k c_{i}\right)^{2}} \frac{d c_{i}}{d t} \\
& \gamma_{i}=1+\frac{k P b_{i}}{\left(1+k c_{i}\right)^{2}} \\
& v_{i} \frac{d C_{i}}{d t}=v_{i} w \text { fract }_{i} \gamma_{i} \frac{d c_{i}}{d t}
\end{aligned}
$$

For the limiting case where binding does not saturate $\left(\mathrm{kc}_{\mathrm{i}}\right.$ $\ll 1), \gamma_{\mathrm{i}}$ in eq. 17 becomes equal to $1 / \mathrm{fw}_{\mathrm{i}}$ and eq. 17 becomes equal to the left hand side of eq. 4 .

The right hand side of eq. 4 has a term that describes the balance between the inflow and outflow of blood from organ i:

$$
\begin{aligned}
& \rho_{B} w \text { fract }_{B} F_{i}\left[\left(c_{B}^{b}+c_{B}\right)-\left(c_{V}^{b}+c_{V}^{i}\right)\right]=\rho_{B} \text { wract }_{B} F_{i}\left[\left(1+\beta_{A}\right) c_{B}-\left(1+\beta_{V}\right) c_{V}\right] \\
& \beta_{A}=\frac{k P b_{B}}{1+k c_{B}} \quad \beta_{V}=\frac{k P b_{B}}{1+k c_{V}}
\end{aligned}
$$

where $\mathrm{c}_{\mathrm{B}}$ and $\mathrm{C}_{\mathrm{v}}^{\mathrm{i}}$ are the free concentration in the blood water entering and leaving organ $\mathrm{i}$, and $\mathrm{c}_{\mathrm{B}}{ }_{\mathrm{B}}$ and $\mathrm{c}_{\mathrm{v}}^{\mathrm{b}}$ are the bound concentrations entering and leaving the organ. As before, $\mathrm{C}_{\mathrm{V}}^{\mathrm{i}}$ can be related to $\mathrm{C}_{\mathrm{B}}$ and the tissue concentration $\mathrm{c}_{\mathrm{i}}$ using fclear $\mathrm{r}_{\mathrm{i}}$ :

$$
c_{V}^{i}=c_{B}-\text { fclear }_{i}\left(c_{B}-c_{i}\right)
$$

Finally, substituting eq. 19 into eq. 18, and equating the right (eq. 17) and left hand sides (eq. 18), the generalization of eq. 4 to include non-linear binding is obtained:

$$
v_{i} w \text { fract }_{i} \gamma_{i} \frac{d c_{i}}{d t}=\rho_{B} w \text { fract }_{B} f_{c l e a r_{i}} F_{i}\left[\kappa_{B} c_{B}-\kappa_{V} c_{i}\right]+\text { cinput }_{i}-Q_{i}
$$

where the new parameters $\kappa_{B}, \kappa_{V}$ and $\gamma$ are defined as follows: For the case where the binding is linear:

$$
\gamma_{i}=\frac{1}{f w_{i}} \quad \kappa_{B}=\kappa_{V}=\frac{1}{f w_{B}}
$$

For the case of non-linear binding (saturation)

(22) $\quad \gamma_{i}=1+\frac{k P b_{i}}{\left(1+k c_{i}\right)^{2}} \quad \kappa_{B}=1+\frac{\beta_{B}}{\text { fclear }_{i}}+\beta_{V}\left(1-\frac{1}{\text { fclear }_{i}}\right) \quad \kappa_{V}=1+\beta_{V}$

There is one other complication introduced by the nonlinear binding. As shown in eq. 3 , the fclear $r_{i}$ that enters eqs. 20-22 depends on $\mathrm{fw}_{\mathrm{B}}$ which, for the non-linear case, is a function of $c_{B}$ (eq. 16). To take this into account, eq. 3 is solved for the value of fclear $\left(c_{B}\right)$ :

$$
\ln \left(1-f_{\text {clear }}\left(c_{B}\right)\right)=\ln \left(1-\text { flear }_{i}\right) \frac{f w_{B}\left(c_{B}\right)}{f w_{B}(0)}=\ln \left(1-f_{\text {clear }}\right) \frac{\left(1+k c_{B}\right)\left(1+k P_{B}\right)}{1+k c_{B}+k P_{B}}=B_{i}
$$

$$
\operatorname{fclear}_{i}\left(c_{B}\right)=1-\exp \left(B_{i}\right)
$$

where fclear $_{i}$ is the clearance in the limit of 0 concentration and is the value the user inputs to PKQuest. This last expression for fclear $_{i}\left(c_{B}\right)$ is substituted for fclear $r_{i}$ in eqs. 20-22.

\section{Modification of lung model to include ventilation-per- fusion mismatch}

The equation in section I for the lung assumed that there was one lung compartment, perfused by $\mathrm{F}_{\mathrm{CO}}$ (cardiac output) with an alveolar volume valv that was ventilated at a rate alvent. This is an idealized case and it is known that even in normal humans the lung is heterogeneous, with different regions receiving different fractions of the cardiac output and the alveolar ventilation. In order to investigate the significance of the ventilation-perfusion mismatch, this feature was incorporated as in option in PKQuest. This is the first time this feature has been used in a PBPK.

As was discussed in detail by Wilson and Beck [54] the standard measurement of the $\dot{V} / \dot{Q}$ distribution does not uniquely characterize the actual ventilation and perfusion heterogeneities to the different regions of the lung and some additional assumptions must be made. The following derivation closely follows the arguments of Wilson 
and Beck [54]. It is assumed that the probability distribution of ventilation and perfusion can be described as a bivariate, uncorrelated, log-normal function:

$$
F(\dot{V}, \dot{Q})=\left[\frac { 1 } { \sqrt { 2 \pi } \sigma _ { V } } \operatorname { e x p } ( - \frac { ( \operatorname { l n } \dot { V } - \mu _ { V } ) ^ { 2 } } { 2 \sigma _ { V } ^ { 2 } } ] \left[\frac{1}{\sqrt{2 \pi} \sigma_{Q}} \exp \left(-\frac{\left(\ln \dot{Q}-\mu_{Q}\right)^{2}}{2 \sigma_{Q}^{2}}\right]\right.\right.
$$

where $\mu_{\mathrm{v}}$ and $\mu_{\mathrm{Q}}$ are the means and $\sigma_{\mathrm{v}}$ and $\sigma_{\mathrm{Q}}$ are the standard deviations of the log normal distribution. The function F returns the fraction of the lung that has ventilation $\dot{V}$ and blood flow $\dot{Q}$ in the interval $d \ln \dot{V}$ and $d \ln$ $\dot{Q}$ centered about $\ln \dot{V}$ and $\ln \dot{Q}$. The function can be normalized by the use of the normalized variables $\mathrm{v}$ and q:

$$
v=\frac{\ln \dot{V}-\mu_{V}}{\sqrt{2} \sigma_{V}} \quad q=\frac{\ln \dot{Q}-\mu_{Q}}{\sqrt{2} \sigma_{Q}}
$$

$$
f(v) f(q) d v d q=\left[\frac{1}{\sqrt{\pi}} e^{-v^{2}}\right]\left[\frac{1}{\sqrt{\pi}} e^{-q^{2}}\right] d v d q
$$

In the implementation in PKQuest, the lung is divided into $(2 \mathrm{~N})^{2}$ equal volume regions by finding the sequence of $v_{i}$ and $q_{i}(i=0 . . N . .2 N)$ centered about 0 such that all integrals of $f(v) d v$ from $v_{i}$ to $v_{i+1}$ have the same value. Then each of the regions $r_{i j}\left(v_{i} . . v_{i+1}, q_{j} . q_{j+1}\right)$ has equal area. Each lung region $r_{i j}$ is assigned a fraction of the total blood flow $\left(\dot{Q}_{i j}\right)$ defined by:

$$
\dot{Q}_{i j}=\int_{q_{i-1}}^{q_{i}} \int_{v_{j-1}}^{v_{j}} \dot{Q}(q) f(v) f(q) d v d q
$$

From the definition of q (eq. 25):

$$
\dot{Q}(q)=e^{\mu_{Q}} e^{\sqrt{2} \sigma_{q} q}=\bar{Q} e^{-\sigma_{q}^{2} / 2} e^{\sqrt{2} \sigma_{q} q}
$$

where, in the second equality in eq. 27, the relation A21 of Beck and Wilson [54] has been substituted for $\exp \left(\mu_{\mathrm{q}}\right)$. Substituting eq. 27 into eq. 26 and integrating, yields the blood flow to the ij region. A similar approach is used to find the ventilation to the ij region.

The overall result of this rather messy procedure can be summarized as follows: The lung is divided into $(2 \mathrm{~N})^{2}$ equal volume regions, each region having a ventilation and blood flow based on the observed log normal distributions. Each region is defined to have the same tissue and alveolar volume. An important assumption that has been used here is that the ventilation and perfusion to each region is uncorrelated. This assumption is consistent with recent direct measurements of regional perfusion and ventilation $[55,56]$.

In the implementation in PKQuest, the number of lung compartments (Nlung) is determined by the parameter N1dim (Nlung $\left.=(2 \mathrm{~N} 1 \mathrm{dim})^{2}\right)$. The default is Nlung $=1$ (indicated by N1dim $=-1$ ). Choosing N1dim = 2, means that the perfusion and ventilation is distributed over 16 lung compartments which should be enough to provide a good test of the importance of perfusion-ventilation mismatch. If Nlung $>1$ then values are required for $\sigma_{\mathrm{v}}$ ( $\operatorname{sig} \_\mathrm{v}$ in PKQuest) and $\sigma_{\mathrm{q}}$ (sig_f). Default values of sig_v and sig_f are included in standardhuman. These default values were obtained using the relation of Wilson and Beck [54] that relates the standard deviation of the $\dot{V} / \dot{Q}$ distribution that is routinely measured $\left(\sigma_{\mathrm{v} / \mathrm{q}}\right)$ to the values of $\sigma_{\mathrm{v}}$ and $\sigma_{\mathrm{q}}$ that are required in eqs. $23-26$ :

$$
\sigma_{V_{A} / Q}^{2}=\sigma_{V}^{2}+\sigma_{Q}^{2}-2 \rho \sigma_{V} \sigma_{Q}
$$

where $\rho$ is the correlation between perfusion and ventilation. The default values in PKQuest are for the case of $\rho=$ 0 , and $\sigma_{\mathrm{v}}=\sigma_{\mathrm{q}}$ and $\sigma_{\mathrm{v} / \mathrm{q}}=0.4$, the normal value for young adult men [57]. The user can input arbitrary values by inputting sig_v and sig_f. This perfusion-ventilation mismatch feature is discussed and tested in the paper describing the application of PKQuest to volatile solutes [2].

\section{Tests of the accuracy of the "find absorption" option of PKQuest}

The "find absorption" option (see II above for details) predicts the PO absorption as a function of time based on the observation of plasma concentration at a set of time points. The following test of this calculation was carried out. It is assumed that the PO input is known and can be described by the following 3-parameter Hill type equation similar to what was found experimentally for propranolol in fig:

(29) Absorption at time $\mathrm{t}=\mathrm{A}\left[\mathrm{t}^{\mathrm{H}} /\left(\mathrm{t}^{\mathrm{H}}+\mathrm{T}^{\mathrm{H}}\right)\right]$ where $\mathrm{A}=80000$ micrograms, $\mathrm{H}=2, \mathrm{~T}=90$

Figure 8 shows the plasma concentration curve generated by PKQuest for this known input. The solid line is the model plasma concentration, and the squares are the values of the plasma concentration, sampled at the same time points as the experimental plasma concentration in the measurements of Olanoff et. al. [5] described in the text. The test of the "find absorption" feature is to see if PKQuest can reproduce the time dependent absorption that was used to generate these points (eq, 29), just using 
these experimental points as inputs. Figure 9 shows the output of PKQuest using the "find absorption" option using the data points (squares) from fig. 8 as input. The value of the total predicted absorption differs from the actual total input by only $0.5 \%$ ( 80505 versus actual 80000 ). The time dependence of the absorption is also quite close to the actual input function (eq. 29), with the Hill exponent $(\mathrm{H})$ differing by $2 \%$ (2.04 versus input 2.0 ) and the half time ( $\mathrm{T}$ ) differing by $4 \%$ (94.63 versus input 90.0 ). This agreement is remarkable, considering the coarse discrete samples of the venous concentration that were used as input to PKQuest.

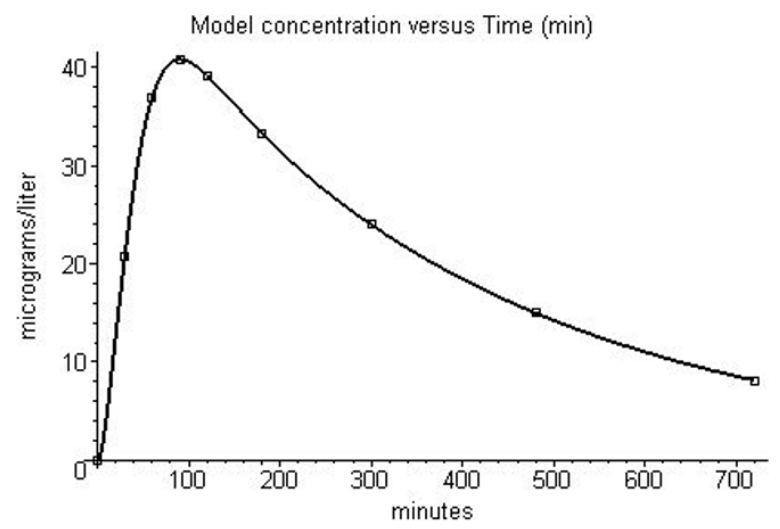

Figure 8

Model venous concentration for the case of an oral input described by eq. 29 . The squares indicate the discrete time points where the venous concentration is sampled. These discrete time points are used as input for the results shown in fig. 9.

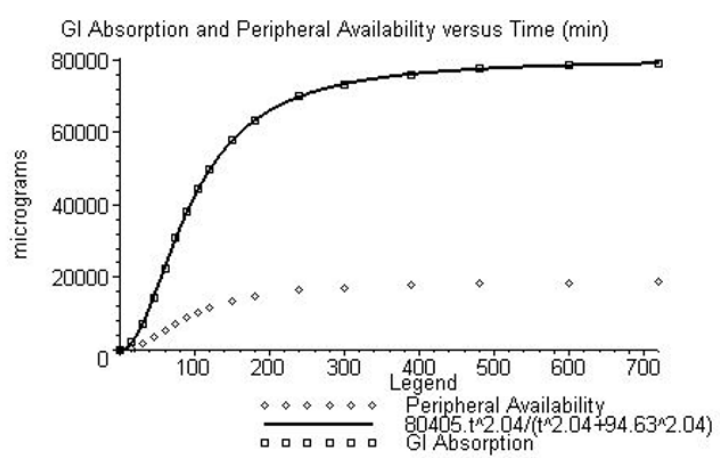

Figure 9

Predicted time course of absorption using the discrete sampled time points (fig. 8). The predicted time course should equal the function (eq. 29) that was used to generate these time points.

\section{Acknowledgements}

I wish to thank Dr Zheng Yang for his careful and detailed review of this manuscript and his many valuable comments and suggestions.

\section{References}

I. Gerlowski LE, Jain RK: Physiologically based pharmacokinetic modeling: principles and applications. J Pharm Sci 1983, 72:1103-27

2. Levitt DG: PKQUEST: Volatile Solutes - Application to Enflurane, Nitrous Oxide, Halothane, Methoxyflurane and Toluene Pharmacokinetics. BMC Anesthesiology 2002, 2:5

3. Levitt DG: PKQUEST: Measurement of Intestinal Absorption and First Pass Metabolism - Application to Human Ethanol Pharmacokinetics. BMC Clinical Pharmacology 2002, 2:4

4. Levitt DG: PKQUEST: Capillary Permeability Limitation and Plasma Protein Binding - Application to Human Inulin, Dicloxacillin and Ceftriaxone Pharmacokinetics. Submitted: BMC Clinical Pharmacology 2002

5. Olanoff LS, Walle T, Cowart TD, Walle UK, Oexmann MJ, Conradi EC: Food effects on propranolol systemic and oral clearance: support for a blood flow hypothesis. Clin Pharmacol Ther 1986, 40:408-14

6. Walle T, Walle UK, Olanoff LS: Quantitative account of propranolol metabolism in urine of normal man. Drug Metab Dispos 1985, 13:204-9

7. Kornhauser DM, Wood AJ, Vestal RE, Wilkinson GR, Branch RA, Shand DG: Biological determinants of propranolol disposition in man. Clin Pharmacol Ther 1978, 23:165-74

8. Pugliese D, Ohnishi K, Tsunoda T, Sabba C, Albano O: Portal hemodynamics after meal in normal subjects and in patients with chronic liver disease studied by echo-Doppler flowmeter. Am J Gastroenterol 1987, 82:1052-6

9. Sasaya S, Yagi H, Yamaguchi M, Kigawa G, Nakano H, Midorikawa T, Nagasaki $H$, Kumada $K$ : Liver function assessed by increased rate of portal venous blood flow after oral intake of glucose. Eur J Surg 2000, 166: 112-8

10. Gaiani S, Bolondi L, Li Bassi S, Santi V, Zironi G, Barbara L: Effect of meal on portal hemodynamics in healthy humans and in patients with chronic liver disease. Hepatology 1989, 9:815-9

11. Lifson N, Levitt DG, Griffen WO Jr, Ellis CJ: Intrahepatic distribution of hepatic blood flow: double-input studies. Am J Physiol 1970, 2 I 8: 1480-8

12. Griffen WO Jr, Levitt DG, Ellis CJ, Lifson N: Intrahepatic distribution of hepatic blood flow: single-input studies. Am J Physiol 1970, 2 I 8: 1474-9

13. Doolette DJ, Upton RN, Grant C: Diffusion-limited, but not perfusion-limited, compartmental models describe cerebral nitrous oxide kinetics at high and low cerebral blood flows. J Pharmacokinet Biopharm 1998, 26:649-72

14. Krejcie TC, Henthorn TK, Niemann CU, Klein C, Gupta DK, Gentry WB, Shanks CA, Avram MJ: Recirculatory pharmacokinetic models of markers of blood, extracellular fluid and total body water administered concomitantly. J Pharmacol Exp Ther 1996, 278: 1050-7

15. Snyder WS, ed: Report of the task group on refereance man. Oxford: Pergamon Press; 1975

16. Williams LR, Leggett RW: Reference values for resting blood flow to organs of man. Clin Phys Physiol Meas 1989, 10:187-217

17. Bischoff KB: Some fundamental considerations of the applications of pharmacokinetics to cancer chemotherapy. Cancer Chemother Rep 1975, 59:777-93

18. Gregersen MI, Nickerson JL: Relation of blood volume and cardiac output to body type. I Applied Physiol 1950, 3:329-4I

19. Wennesland R, Brown E, Hopper J, Hodges JL, Guttentag OE, Scott KG, Tucker IN, Bradley B: Red cell, plasma and blood volume in health men measured by radiochromium cell tagging and hematocrit. J Clin Invest 1959, 38: I065-76

20. Pierson RN Jr, Wang J, Colt EW, Neumann P: Body composition measurements in normal man: the potassium, sodium, sulfate and tritium spaces in $\mathbf{5 8}$ adults. J Chronic Dis 1982, 35:419-28

21. Chumlea WC, Guo SS, Zeller CM, Reo NV, Baumgartner RN, Garry PJ, Wang J, Pierson RN Jr, Heymsfield SB, Siervogel RM: Total body water reference values and prediction equations for adults. Kidney Int 200I, 59:2250-8 
22. Schloerb PR, Friis-Hansen BJ, Edelman IS, Solomon AK, Moore FD: The measurement of total body water in the human subject by deuterium oxide dilution. J Clin Invest I950, 29:1296-3 I0

23. Jonsson F, Johanson G: Physiologically Based Modeling of the Inhalation Kinetics of Styrene in Humans Using a Bayesian Population Approach. Toxicol Appl Pharmacol 2002, 1 79:35-49

24. Fagan TC, Walle T, Walle UK, Conradi EC, Harmon G, Gaffney TE: Early kinetics of intravenous propranolol. Br J Clin Pharmacol 1982, | 3:57|-4

25. Takahashi H, Ogata H, Warabioka R, Kashiwada K, Ohira M, Someya $\mathrm{K}$ : Decreased absorption as a possible cause for the lower bioavailability of a sustained-release propranolol. J Pharm Sci 1990, 79:212-5

26. Ingber L, Rosen B: Genetic algorithms and very fast simulated reannealing: A comparison. Mathamatical and Computer Modeling 1992, I 6:87-100

27. Wensheng $C$, Xueguang S: A fast annealing evolutionary algorithm for global optimization. Journal Computer Chemistry 2002, 23:427-435

28. Press WH, Teukolsky SA, Vetterling WT, Flannery BP: Numerical Recipes in C Cambridge: Cambridge University Press 1992

29. Levitt MD, Levitt DG: Appropriate use and misuse of blood concentration measurements to quantitate first-pass metabolism. J Lab Clin Med 2000, I 36:275-80

30. Naranjo CA, Sellers EM, Khouw V: Unaltered serum propranolol binding by meal-induced variations in fatty acids. $\mathrm{Br}$ J Clin Pharmacol 1982, 13:575-6

31. Schneck DW, Pritchard JF, Hayes AH Jr: Studies on the uptake and binding of propranolol by rat tissues. J Pharmacol Exp Ther 1977, 203:62।-9

32. Paxton JW, Calder RL: Propranolol binding in serum: comparison of methods and investigation of effects of drug concentration, pH, and temperature. J Pharmacol Methods 1983, I 0: I- I I

33. Yata N, Toyoda T, Murakami T, Nishiura A, Higashi Y: Phosphatidylserine as a determinant for the tissue distribution of weakly basic drugs in rats. Pharm Res 1990, 7:1019-25

34. Cheymol G, Poirier JM, Carrupt PA, Testa B, Weissenburger J, Levron JC, Snoeck E: Pharmacokinetics of beta-adrenoceptor blockers in obese and normal volunteers. $\mathrm{Br}$ J Clin Pharmacol 1997, 43:563-70

35. Silber BM, Holford NH, Riegelman S: Dose-dependent elimination of propranolol and its major metabolites in humans. I Pharm Sci 1983, 72:725-32

36. Borgstrom L, Johansson CG, Larsson H, Lenander R: Pharmacokinetics of propranolol. J Pharmacokinet Biopharm I98I, 9:4I9-29

37. Mould GP, Clough J, Morris BA, Stout G, Marks V: A propranolol radioimmunoassay and its use in the study of its pharmacokinetics following low doses. Biopharm Drug Dispos 1981, 2:49-57

38. Olanoff LS, Walle T, Walle UK, Cowart TD, Gaffney TE: Stereoselective clearance and distribution of intravenous propranolol. Clin Pharmacol Ther 1984, 35:755-6I

39. Jackman GP, McLean AJ, Jennings GL, Bobik A: No stereoselective first-pass hepatic extraction of propranolol. Clin Pharmacol Ther 1981, 30:291-6

40. Watari N, Benet LZ: Determination of mean input time, mean residence time, and steady-state volume of distribution with multiple drug inputs. J Pharmacokinet Biopharm 1989, I 7:593-9

41. Orlando R, Floreani M, Padrini R, Palatini P: Determination of inulin clearance by bolus intravenous injection in healthy subjects and ascitic patients: equivalence of systemic and renal clearances as glomerular filtration markers. $\mathrm{Br}$ J Clin Pharmacol 1998, 46:605-9

42. Csanady GA, Mendrala AL, Nolan RJ, Filser JG: A physiologic pharmacokinetic model for styrene and styrene-7,8-oxide in mouse, rat and man. Arch Toxicol 1994, 68:143-57

43. Fisher JW, Whittaker TA, Taylor DH, Clewell HJ 3rd, Andersen ME: Physiologically based pharmacokinetic modeling of the pregnant rat: a multiroute exposure model for trichloroethylene and its metabolite, trichloroacetic acid. Toxicol Appl Pharmacol 1989, 99:395-414

44. Thongnopnua P, Massarella JW, Zimmerman CL: The pharmacokinetics of etretinate and its metabolites in the dog. Drug Metab Dispos 1989, I 7:473-80

45. Staats DA, Fisher JW, Connolly RB: Gastrointestinal absorption of xenobiotics in physiologically based pharmacokinetic models. A two-compartment description. Drug Metab Dispos 1991, 19:144-8

46. Semino G, Lilly P, Andersen ME: A pharmacokinetic model describing pulsatile uptake of orally - administered carbon tetrachloride. Toxicology 1997, I I 7:25-33

47. Veng-Pedersen P: Linear and nonlinear system approaches in pharmacokinetics: how much do they have to offer? I. General considerations. J Pharmacokinet Biopharm 1988, 16:413-72

48. Gan G, Cartier LL, Huang Y, Yang Z, Sawchuk RJ: Intestinal absorption and presystemic elimination of the prokinetic agent, EM574, in the rabbit. J Pharm Sci 2002, $91: 217-28$

49. Pithavala YK, Soria I, Zimmerman CL: Use of the deconvolution principle in the estimation of absorption and pre-systemic intestinal elimination of drugs. Drug Metab Dispos 1997, 25: 1260-

50. Melander A, Danielson K, Schersten B, Wahlin E: Enhancement of the bioavailability of propranolol and metoprolol by food. Clin Pharmacol Ther 1977, 22:108-12

5I. Walle T, Fagan TC, Walle UK, Oexmann MJ, Conradi EC, Gaffney TE: Food-induced increase in propranolol bioavailability - relationship to protein and effects on metabolites. Clin Pharmacol Ther 1981, 30:790-5

52. DiPadova C, Worner TM, Julkunen RJ, Lieber CS: Effects of fasting and chronic alcohol consumption on the first-pass metabolism of ethanol. Gastroenterology 1987, 92: I 169-73

53. Tsuji A, Yoshikawa T, Nishide K, Minami H, Kimura M, Nakashima E, Terasaki T, Miyamoto E, Nightingale CH, Yamana T: Physiologically based pharmacokinetic model for beta-lactam antibiotics I: Tissue distribution and elimination in rats. J Pharm Sci I983, 72:1239-52

54. Wilson TA, Beck KC: Contributions of ventilation and perfusion inhomogeneities to the VA/Q distribution. J Appl Physiol 1992, 72:2298-304

55. Gerbino AJ, McKinney S, Glenny RW: Correlation between ventilation and perfusion determines VA/Q heterogeneity in endotoxemia. J Appl Physiol 2000, 88:1933-42

56. Treppo S, Mijailovich SM, Venegas JG: Contributions of pulmonary perfusion and ventilation to heterogeneity in $\mathrm{V}(\mathrm{A}) / \mathbf{Q}$ measured by PET. I Appl Physiol 1997, 82: I 163-76

57. Wagner PD, Laravuso RB, Uhl RR, West JB: Continuous distributions of ventilation-perfusion ratios in normal subjects breathing air and 100 per cent 02. J Clin Invest 1974, 54:54-68

\section{Pre-publication history}

The pre-publication history for this paper can be accessed here:

http://www.biomedcentral.com/1472-6904/2/5/prepub 
Publish with BioMed Central and every scientist can read your work free of charge

"BioMedcentral will be the most significant development for disseminating the results of biomedical research in our lifetime." Paul Nurse, Director-General, Imperial Cancer Research Fund

Publish with BMC and your research papers will be:

- available free of charge to the entire biomedical community

- peer reviewed and published immediately upon acceptance

- cited in PubMed and archived on PubMed Central

- yours - you keep the copyright

BioMedcentral.com editorial@biomedcentral.com 